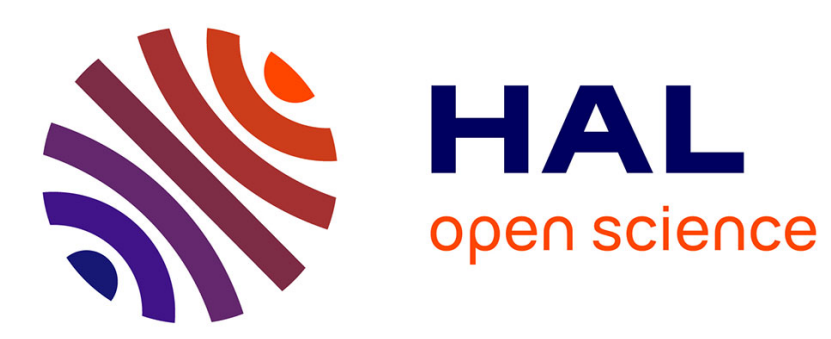

\title{
Inference of the Bottom Properties in Shallow Ice Approximation Models
}

\author{
Jerome Monnier, P.-E Des Boscs
}

\section{To cite this version:}

Jerome Monnier, P.-E Des Boscs. Inference of the Bottom Properties in Shallow Ice Approximation Models. Inverse Problems, 2017, 33 (11), pp.115001. hal-01545171v2

\section{HAL Id: hal-01545171 \\ https://hal.science/hal-01545171v2}

Submitted on 18 Feb 2019

HAL is a multi-disciplinary open access archive for the deposit and dissemination of scientific research documents, whether they are published or not. The documents may come from teaching and research institutions in France or abroad, or from public or private research centers.
L'archive ouverte pluridisciplinaire HAL, est destinée au dépôt et à la diffusion de documents scientifiques de niveau recherche, publiés ou non, émanant des établissements d'enseignement et de recherche français ou étrangers, des laboratoires publics ou privés. 


\title{
Inference of the Bottom Properties in Shallow Ice Approximation Models
}

\author{
J. Monnier, P.-E. des Boscs
}

INSA \& Mathematics Institute of Toulouse, F-31077 Toulouse cedex 4, France.

5 jerome.monnier@insa-toulouse.fr

This study proposes a new inverse method to infer the bottom topography and the friction coefficient in the Shallow Ice Approximation (SIA) model (lubrication type models for generalized Newtonian fluid flows). The method is based on the definition of three sub-regimes and an a-priori slip ratio law. Next, explicit calculations provide a first depth estimation for each sub-regime, and this first estimation can be improved by solving an elliptic linear-quadratic optimal control problem (variational assimilation of the surface measurements e.g. by satellite). The friction field is a by-product of the depth inverse method, it can be explicitly deduced. The numerical results performed on multi-regime numerical flows demonstrate the robustness of the method even in presence of uncertain surface measurements and independently of the depth measurement locations (on contrary if inverting the regularized depth-averaged mass equation only). Moreover the few derived depth estimations make possible to determine the adequate slope scale in the SIA models.

Keywords. Shallow flow, Shallow Ice Approximation, topography inference, friction, data assimilation, glaciers.

\section{Introduction}

The knowledge of the bottom topography is a basic step to set up a numerical flow model; however this crucial data may be unknown or difficult to acquire especially in geophysics. Inverse methods to infer the topography, and potentially the basal slipperiness, are then the only alternative (for a review on inverse methods in free surface flows see e.g. [41]). The bottom topography knowledge combined with the top surface measurements gives straightforwardly a volume - mass estimation; the next important quantity to estimate is the flux. For ice flows, these estimations are important in the context of global warming and sea level rise.

An extra difficulty of shallow flow inverse modeling is the following: even if the free surface presents very small and smooth variations, the bottom topography may not. Indeed, the shallow fluid flows (even newtonian) act as low-band filters: the bed variations are filtered by the flow. The filtering features depend on the sliding amount at bottom, see $[12,13,25,24]$ for detailed analysis applied to generalized newtonian or ice flows.

In the case of no-slip at bottom, the inference of the bottom properties is limited to the topography only. Then the difficulty of the corresponding inverse problem depends on the observation availability. In the case of sliding at bottom (or equivalently finite friction), the inverse problem becomes much more difficult since the unknown becomes the (topography, friction) pair. Then a challenging goal is to separate the signature of these two different bottom properties (topography and friction), usually given surface observations - measurements. Under some flow conditions, these two features can lead to equivalent bottom conditions hence making the inference of the property pair particularly challenging, see e.g. $[16,5,7]$ in the Newtonian case (shallow water flows) and $[13,25]$ in the power-law / Glen-Nye's law case.

The direct measurements of ice thickness, for example along a track using airborne radio-echo sounding, are time-consuming, expensive and provide very sparse measurements only. Also radio-echo sounding can be inaccurate due to water beneath the glaciers e.g. in southern Greenland. The current bedrock maps for Greenland [2] and Antarctica [35] are available at $1 \mathrm{~km}$ resolution from measurement surveys plus Kriging interpolation techniques (with simple relations based on the surface slope). But the uncertainty between the measurements still prevents to set up high resolution dynamic ice flow models; and despite the important measurement campaigns, e.g. NASA Operation Ice Bridge. In other respect, various satellites provide quite accurate and frequent $(\approx 10$ days revisits $)$ measurements of the ice sheet surfaces: altimeters provide the surface elevation $H(x, t)$ at $\approx+/-10-30 \mathrm{~cm}$ for $1 \mathrm{~km}^{2}$ pixels, while radar interferometers (InSar) provide the surface velocity $\mathbf{u}_{H}$ (more or less accurately depending on its magnitude, see e.g. [39]). For non ice-sheet glaciers, e.g. Arctic, Alaska, Patagonia or High Mountains, in-situ measurements are usually the surface elevation while velocity measurements are less usual. 
A great challenge is to fill up the gaps between the reliable depth measurements and to determine an effective slipperiness (modeling basal conditions e.g. mix of rocks and mud or subglacial hydrology networks). This goal can be partially reached by developing inverse methods combining all available information.

Let us introduce some notations, the standard depth-averaged mass equation and the classical Shallow Ice Approximation (SIA) equation (standard lubrication approximation). The surface flow elevation is denoted by $H$, the bed elevation is denoted by $b$ and the ice depth (or thickness) is defined by $h, h=(H-b)$, see Fig. 1 .

Then the depth-averaged mass equation reads:

$$
\operatorname{div}(h \overline{\mathbf{u}})=a-\partial_{t} h \equiv \dot{a}
$$

where $\overline{\mathbf{u}}$ is the depth-averaged velocity and $a$ is the mass balance source term. The Shallow Ice Approximation (SIA) (lubrication type equation) is obtained by injecting the velocity expression $\overline{\mathbf{u}}$ (which can be derived explicitly) into the mass equation above, see e.g. $[10,6]$. This gives:

$$
-\operatorname{div}_{x y}\left(\alpha h^{5}|\nabla H|^{2} \nabla H\right)=\dot{a}
$$

where $\alpha$ is a coefficient depending on the ice properties. This SIA model version is the most employed one, no slip is imposed at bottom $\left(\mathbf{u}_{b}=0\right)$ hence no friction coefficient $C$ appears in the equation. More details on the shallow flow model derivations are presented in the manuscript.

The inference of the bottom properties (usually the topography only) by inverting a ice model has been addressed in numerous studies. First let us mention the pioneer article [38]. The ice depth $h$ or/and the effective mass balance $\dot{a}$ are inferred from the depth-averaged mass equation combined with some surface elevation data $H^{o b s}$, surface velocity data $\mathbf{u}_{H}^{o b s}$ and depth measurements $h^{o b s}$ available along flight tracks over the Columbia glacier in Alaska. In the mass equation, the mean velocity is empirically related to the surface velocity by a coefficient $\gamma, \gamma=\overline{\mathbf{u}} / \mathbf{u}_{H}$. (Remark that given the power-law exponent of the fluid, $\gamma$ defines the vertical velocity profile; somehow this spatially-distributed coefficient $\gamma$ provides a velocity model, replacing the missing momentum equation). Next a quadratic cost function measuring the differences between the model output and 70 the surface velocity measurements is defined. Finally the first-order optimality condition (gradient of the cost function vanishes) is numerically solved by the Newton algorithm. Note that this pioneering study has been carried out before the classical use of the optimal control techniques in data assimilation problems, see e.g. [22], and before the acquisition of rich satellite measurements. In [44], the authors employ the explicit depth expression in function of the SIA equation flux: $h^{5}=\frac{1}{\alpha} \frac{\Phi}{\left.\nabla H\right|^{3}}$, where the flux $\Phi$ is estimated by inverting the divergence operator (given $\dot{a}$ ). An other pioneer study is [43] since it does not address the topography inference only but the (topography $b$, friction $C$ ) pair inference. This is done for fast-flowing ice-streams (unsheared flows) hence not modeled by the SIA equation. The inference is based on the linearized model equation and the analysis of the transmission of flow disturbances through the ice thickness and from surface observations (elevation,velocity). More recently [31,34] have elaborated a combination of efficient computational algorithms based on a Variational Data Assimilation (VDA), solving the same equations than [38]. The resulting algorithm combined with multi-sources and heterogeneous data sets leads to rich bed maps at $\approx 300 \mathrm{~m}$ resolution in the challenging South Greenland regions where airborne radar sounding are inaccurate, see [32, 33]. However this method has few drawbacks since it relies on a transport equation, hyperbolic first order. Indeed the equation feature limits the inversion capabilities at locations downstream the depth measurements. In other words, data are required at the characteristic inflow locations. Moreover the hyperbolic feature of the model leads to error propagations e.g. the surface measurement errors. In [42, 15] the authors reconstruct the surface elevation $H$ and the bed elevation $b$ from the surface velocity $\mathbf{u}_{H}$ and depth measurements given at upstream again. The inversions are based on the traditional SIA equation and velocity expression i.e. with no-slip at bottom only. This limits the applicatibility of the approach. Similarly [26] infers the topography of non-sliding mountain glaciers from the surface elevation data $H$ (and mass-balance $a$ ). Again, since the direct model is a non-linear transport equation (the inverse problem aims at inverting the divergence operator), the authors solve a pseudo-time dependent problem and introduce artificial diffusion to regularize the first order operator. Let us point out that the introduction of artificial diffusion to invert the divergence operator presents serious drawbacks: the inverse problem is not the original one and the computed solution is fully dependent on the arization parameter. Also, the inversion is highly unstable or even numerically impossible, depending on the locations of the measurements at boundaries. Roughly, only data in the vicinity of a characteristics root can be inferred (roughly for a given flow line, this corresponds to the inflow data). In the unsteady case, the authors of [26] point out that smoothing the surface data is mandatory, then the computed solution is fully dependent on this additional regularization parameter. Next the same authors developed a so-called "shape ptimization" approach, see [27] (actually it is a parameter identification method since it aims at identifying the model parameter $b$ while the domain shape is fixed). The problem is formulated as a classical optimal control problem solved by VDA, leading to a more robust than the algorithms presented in [26]. Let us point that such a straighforward "blind" VDA may be unable to infer the bottom properties in presence of the additional 
unknown: the spatially distributed friction parameter $C(x, t)$. Indeed in this case, equifinality issues can appear as demonstrated in different ways and in different contexts in $[11,12]$. Other studies address the topography inference but either in 1D only (following flow-lines) and/or based on empirical flux estimations, see e.g. [17].

All the aforementioned existing methods, excepted [43], are either based on the regularization of a transport equation (hence presenting drawbacks and limitations) and/or consider the inference of the topography only. The inverse method of [43] is based on the perturbation theory developed in [11] hence small variations of the inferred quantity $(b, C)$ only can be inferred, moreover for fast ice streams only (in this case, the bed-to-surface transfer function is much less filtered compared to sheared flows).

The present study aims at infering the complete bottom properties i.e. the (topography b, friction $C$ ) pair in a multi-regime shallow ice flow (hence presenting local and extremely stiff variations of the friction parameter $C$ ) without any artificial regularization nor particular measurement location requirements (like it is the case if inverting the first order divergence operator). Since the model considered is the SIA model with potential slip at bottom (model so-called extended SIA), the domain of validity of the method ranges from fully sheared to mildly sheared flows hence valid for a large panel of observed ice flows (however this excludes the fast ice-streams).

The present method guideline is to consider intrinsically invertible equations only (typically diffusive / parabolic-elliptic second order equations and not first order equations) and to consider robust, well-posed optimal control problems only. The goal has been reached by basing the inverse method on a combination of explicit field expressions of the xSIA equation and an intermediate linear-quadratic optimal control problem (hence well-posed); the latter is classically solved by VDA.

The required measurements are the surface elevation $H$ and the surface velocity norm $\left|\mathbf{u}_{H}\right|$ plus some in-situ depth measurements. The latter can be located anywhere relatively to the stream lines. The strengths of the resulting invertion method are the following:

i) A high robustness, including in presence of uncertain surface measurements and whatever the depth measurements location;

ii) A large domain of validity in terms of flow regimes: from fully sheared to mildly sheared flows. Thus it can be applied to the great majority of the ice-sheet surfaces (corresponding to the accumulation areas) and high-mountains glaciers; but this excludes the fast ice-streams. (Recall that for fast ice-stream in coastal ice sheet areas, the depth-mass equation inversion can be efficient, [31, 34, 33]).

iii) A capability to infer separately the depth from the spatially distributed friction coefficient $C$. The inference of $C$ becomes a by-product of the depth inference. This feature is important since equifinality issues potentially occur when identifying the pair (topography, friction). Also the friction coefficient is one of the greatest uncertain parameter in geophysical shallow flow models, its values vary within few orders of magnitudes and its estimation by expert assessment is generally impossible.

Finally a by-product of the present analysis is a methodology to define consistent slope scales in SIA models.

Since based on shallow flow equations, this inverse method remains affordable even for large computational domains (e.g. the whole ice-sheets); this is particular true if the VDA process is implemented in parallel like it can be done in DassFlow [29] or ISSM [21] for example. The present numerical results have been performed by using the Fenics Python library [1, 23].

The weakness of the method is its relative inaccuracy in the narrow regime transition areas like canyon margins. This gives local error peaks (of 20-30\% approx.) corresponding to the stiff variations of the inferred quantities (variations extremely local and of few orders of magnitude like the friction coefficient).

The outline of the article is as follows. In Section 2, the SIA model is recalled, explicit field expressions are re-derived. The domain of validity of the model in terms of basal friction amount (or equivalently in terms of the slip ratio $R_{s}$ ) is discussed; this leads to the definition of three sub-regimes corresponding to different slip ratio range values. Section 3 describes the inverse problem and the inference method. For each sub-regime, an explicit expression of the depth $h$ is derived; all of them depend on a unique observational surface term denoted $\mathcal{Q}_{H}$. For two sub-regimes, the depth expression depends on the "diffusivity" $\eta$ of the xSIA equation. This diffusivity is numerically computed by VDA. Finally, the friction coefficient value $C$ can be explicitly deduced. Next, the complete method is assessed on academic test cases presenting the complete range of flow regimes with stiff transition areas (hence presenting many difficulties of real-world flows). The multi-regime academic test case is constructed in Section 4. A method to define an a-priori slip ratio map next an already good first depth estimation is developed in Section 5. Section 6 aims at analysing the numerical results in the case of downstream depth measurements, both for perfect and uncertain surface measurements. Various sensitivity analysis (explicit and numerical ones) are highlighted and commented. An extra difficult test case based on lateral depth measurements only (measurements quasi parallel to a flow line, moreover within a mono-regime area) is analysed in Section 7. Finally, it turns out that the few depth estimations aforementioned make possible to determine the correct slope scale in a SIA model. This is explained in Section 8 and it is illustrated for 1D flows. The conclusion proposes few perspectives of this preliminary study. 


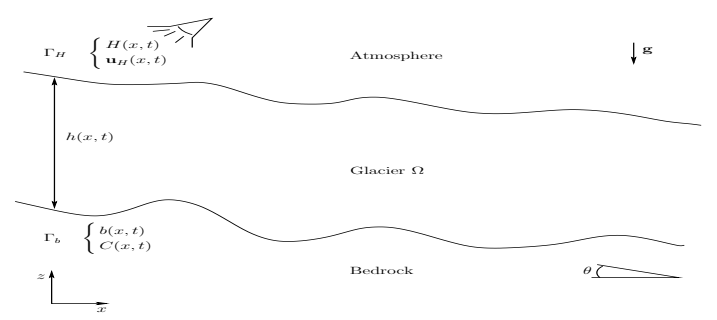

Figure 1: Notations. A shallow ice flow ( $\mathrm{x}-\mathrm{z}$ view) along an inclined mean slope, with surface observations.

\section{The direct model}

In this section, the classical Shallow Ice Approximation (SIA) velocity expressions and resulting equations are recalled, see [18, 30], also e.g. [6] chapter 10.2; also some various explicit field expressions useful in the forthcoming inverse method are derived. The SIA model derives from an asymptotical calculations of the free surface (non-linear) Stokes equations with respect to the geometrical ratio $\varepsilon=\frac{H^{*}}{L^{*}}$; with $H^{*}$ a characteric flow depth and $L^{*}$ a characteric flow length. The basic assumption states that the flow, thin geometry, is sheared; in other words, the normal stress components are negligible. The SIA equations are first order in $\varepsilon$. The expression "SIA model" denotes indistinctly the explicit velocity expressions (derived from the momentum equations only) and the corresponding lubrication type equation (derived from the depth-averaged mass equation); also whatever if trhe flow is fully sheared flow (with no slip at bottom) or if it is partly sheared (with friction condition at bottom). For a sake of precision, in the present article the SIA model including slip amount at bottom is called extended SIA (xSIA). In [4], it is formally demonstrated that the xSIA model remains valid for a friction coefficient $C \simeq \mathcal{O}(1)$ hence clarifying the xSIA domain of validity. This legitimates the few sub-regimes considered in next section. (In [4], the xSIA equation is a particular case of more general shallow flow models, see Section 11 of this reference). In other respect in the literature, see e.g. [6] chapter 10.2 or [10]chapter 5.4., the xSIA equation is derived in the mean slope coordinate system (frequently in the horizontal-vertical coordinate system). Nevertheless it is demonstrated in [4] that the same expressions and equation remain valid in a more general coordinate system: the Prandlt coordinate system [36, 37]. This makes possible to apply the model to any bottom shape i.e. without any clear mean slope, like those observed in some high mountains glaciers for example. In summary, the xSIA equation and field expressions derived below are valid for any coordinate system (here presented in the mean slope coordinate system for the sake of clarity), non-isothermal flows and flows from fully sheared (no slip, $C=0)$ to mildly sheared $(C \simeq \mathcal{O}(1)$ ).

\section{Notations and basic relations}

We denote by $H$ the fluid elevation, $b$ the topography elevation and $h=(H-b)$ the fluid depth. It is assumed that the geometry presents a "mean slope" in the $(x, y)$-plane which can be described by the angle $\theta$, Fig. 1.

The depth averaged velocity $\overline{\mathbf{u}}$ and the discharge $\mathbf{q}$ are defined by: $\overline{\mathbf{u}}=\frac{1}{h} \int_{b}^{H} \mathbf{u}(z) d z$ and $\mathbf{q}=h \overline{\mathbf{u}}=h(\bar{u}, \bar{v})$.

The depth averaged mass equation reads: $\partial_{t} h+\operatorname{div}_{x y}(\mathbf{q})=a$, with $a$ the mass balance (between accumulation and ablation).

We define $\mathcal{S}=|\mathbf{S}|=|\nabla H|$ the free surface slope norm and $\bar{\rho}=(\rho g \cos \theta)^{q}, \rho$ being the fluid density, $g$ the gravity amplitude, $q$ the power-law exponent of the rheology law. The depth-integrated SIA velocity expressions reads: $\mathbf{u}(\mathbf{x}, z)=(u, v)(\mathbf{x}, z)=\mathbf{u}_{b}+2 \bar{\rho} \mathcal{S}^{q-1} \mathbf{S} \int_{b}^{z} A(H-\xi)^{q} d \xi$, see e.g. [6] chapter 10.2 (or [10] chapter 5.4 in the horizontal-vertical coordinate system, $\theta=0$ ) . For glaciers, the usual exponent value is $q=3$.

On the bottom boundary $\Gamma_{b}$, a friction condition is imposed; it is classically described by a power-law with its exponent equal to the rheology law's one (exponent denoted $q$ ). The friction law models the interface between the fluid (e.g. ice) and the bedrock as a viscous boundary layer. Then the basal velocity reads: $\mathbf{u}_{b}=C \bar{\rho} h^{q}|\mathbf{S}|^{q-1} \mathbf{S}$. Finally the longitudinal velocity reads:

$$
\mathbf{u}(\mathbf{x}, z)=(u, v)(\mathbf{x}, z)=\bar{\rho}\left[C h^{q}+2 \int_{b}^{z} A(H-\xi)^{q} d \xi\right] \mathcal{S}^{q-1} \mathbf{S}
$$

The coefficient $C$ is the slip coefficient. If $C \rightarrow 0$, the no-slip condition (adherence) is imposed; on the contrary if $C \rightarrow \infty$ a pure slip condition (vanishing friction) is imposed. Nevertheless, to remain within the SIA model validity, the slip coefficient $C$ has to vary from 0 to $\mathcal{O}(1)$ at most, see e.g. [4] for a detailed discussion and analysis. 


\section{Explicit expressions (non-constant rate factor case)}

The rate factor $A$ depends a-priori on the space variables $(\mathbf{x}, z)$ (hence in particular on the depth variable $z$ ). In glaciology, $A$ depends on the (non-uniform) temperature field. In the forthcoming analytico-numerical inversion method, explicit expressions of $\overline{\mathbf{u}}$ and $\mathbf{u}_{H}$ are required (including in the case $A$ non constant). Then, we introduce $\bar{A}$ independent of $z$ defined by: $\bar{A} \cdot \int_{b}^{H} \int_{b}^{z}(H-\xi)^{q} d \xi d z=\int_{b}^{H} \int_{b}^{z} A(H-\xi)^{q} d \xi d z$. Hence:

$$
\bar{A}(\mathbf{x}, t)=\frac{q+2}{h^{q+2}} \int_{b}^{H} \int_{b}^{z} A(H-\xi)^{q} d \xi d z \text { for all }(\mathbf{x}, t)
$$

Given $A(z)$, the quantity $\bar{A}$ can be computed numerically for each $(\mathbf{x}, t)$. Then the depth averaged velocity reads:

$$
\overline{\mathbf{u}}(\mathbf{x}, t)=\bar{\rho}\left[C+\frac{2 \bar{A}}{(q+2)} h\right] h^{q} \mathcal{S}^{q-1} \mathbf{S}(\mathbf{x}, t)
$$

Similarly, an explicit expression of the surface velocity $\mathbf{u}_{H}$ can be derived. To do so, the scalar value $\underline{A}$ is defined as follows: $\int_{b}^{H} A(H-\xi)^{q} d \xi=\underline{A} \int_{b}^{H}(H-\xi)^{q} d \xi$. Hence:

$$
\underline{A}(\mathbf{x}, t)=\frac{q+1}{h^{q+1}} \int_{b}^{H} A(H-\xi)^{q} d \xi \text { for all }(\mathbf{x}, t)
$$

Then the surface velocity $\mathbf{u}_{H}$ reads:

$$
\mathbf{u}_{H}(\mathbf{x}, t)=\bar{\rho}\left[C+\frac{2 \underline{A}}{(q+1)} h\right] h^{q} \mathcal{S}^{q-1} \mathbf{S}(\mathbf{x}, t)=\mathbf{u}_{b}+\frac{2 \bar{\rho} \underline{A}}{(q+1)} h^{q+1} \mathcal{S}^{q-1} \mathbf{S}(\mathbf{x}, t)
$$

Let us point out that in the case $A$ independent of $z$ (e.g.isothermal glacier flows), $\bar{A}=\underline{A}=A$.

\section{Slip ratio expression}

We define the slip ratio as follows: $R_{s}=\frac{\left|\mathbf{u}_{H}\right|-\left|\mathbf{u}_{b}\right|}{\left|\mathbf{u}_{H}\right|}=1-\frac{\left|\mathbf{u}_{b}\right|}{\left|\mathbf{u}_{H}\right|}$. Then its explicit expression in function of $C$ and $h$ reads:

$$
R_{s}=1-\frac{C}{\left[C+\frac{2 A}{(q+1)} h\right]}=\frac{\frac{2 A}{(q+1)} h}{\left[C+\frac{2 A}{(q+1)} h\right]}
$$

The slip ratio $R_{s}$ equals 1 for fully sheared flow (sub-regime 1 ) and equals 0 for plug like flow. The case $R_{s}=0$ is out of the validity range of the SIA model since the scalings assumed to derive the velocity expressions are not valid anymore. The present SIA equations are a-priori valid up to a slip ratio $R_{s} \approx 0.5$ since the basic scaling done in the SIA equations make possible the asymptotic derivations up to a balance between $C$ and the term $\frac{2 A}{(q+1)} h$, see $[40,4]$ for detailed discussions. Note that $R_{s}=0.5$ is equivalent to the equality: $C=\frac{2 A}{(q+1)} h$.

\section{The xSIA equation}

By injecting the discharge expression $\overline{\mathbf{q}}=h \overline{\mathbf{u}}$ with (3) into the free surface dynamics equation, the one-equation

model (lubrication type) is obtained: $\partial_{t} h-\bar{\rho} d i v_{x y}\left(\left[C+\frac{2 \bar{A}}{(q+2)} h\right] h^{q+1} \mathcal{S}^{q-1} \nabla H\right)=a$. Recall that: $\mathcal{S}=|\nabla H|$, $q \geq 1$. This is the xSIA equation. In a glacier modeling context, uncertainties on the mass source term $a$ (modeling the ablation / deposition at the surface) may be of the same order than the time variation $\partial_{t} h$. (This depends of course on the considered time scale and/or the flow). Then it is classical to consider the effective source term: $\dot{a}=-\partial_{t} h+a$. Then the steady-state version of the xSIA equation reads:

$$
-\bar{\rho} \operatorname{div}_{x y}\left(\eta(C, h) \mathcal{S}^{q-1} \nabla H\right)=\dot{a} \text { with } \eta(C, h)=\left[C+\frac{2 \bar{A}}{(q+2)} h\right] h^{q+1}
$$

The term $\eta$ denotes the "effective diffusivity" of the model. The xSIA equation is non linear, elliptic. Its has to be closed with boundary conditions on $H$ (Dirichlet conditions or mixed ones). Note that if the diffusivity $\eta(C, h)$ is given, if the surface slope $\mathcal{S}$ is given too (from surface measurements) then (7) is a gentle linear elliptic equation. Nevertheless, its is well-known and easily verified that its solution $H$ is highly sensitive to the definition scale of the slopes $\mathcal{S}$. This critical feature is addressed in the last section. 


\section{Inference of the (topography, friction) pair from the surface mea- surements}

The direct problem reads as follows: given the basal properties (topography, friction coefficient) $(b, C)$, find $H$ solution of (7) accompanied with boundary conditions. Then the depth $h=(H-b)$ is straightforwardly deduced. The inverse problem reads as follows: given the surface observations $\left(\mathrm{H}, \mathcal{S}, \mathbf{u}_{H}\right)$, find the pair $(h, C)$.

(Note that the slope value $\mathcal{S}$ measured by altimetry may be more accurate than the gradient of the measured elevation $H$ ).

In this section, three depth estimations are derived. Each estimation correspond to a particular sub-regime defined in terms of slip ratio. These sub-regimes correspond to: fully sheared flows (sub-regime 1), mildly sheared flows (sub-regime 2) and weakly sheared flows (sub-regime 3). In the sub-regime 1 case, the depth estimation is directly obtained from an unique observational term $\mathcal{Q}_{H}\left(\mathcal{Q}_{H}\right.$ depending on the surface slope values $\mathcal{S}$ and the surface velocity norms $\left|\mathbf{u}_{H}\right|$ ). In the sub-regime 3 case, the depth estimation is explicit but in function of the diffusivity $\eta$ of the xSIA equation (7) too. However $\eta$ can be inferred by the Variational Data Assimilation process described in the last subsection (well-posed linear-quadratic control problem). The intermediate regime (sub-regime 2) is the most general one since it includes the two others. In this case, the depth can be inferred as a root of a polynomial depending on the diffusivity $\eta$ (and the observational term $\mathcal{Q}_{H}$ ). Finally the friction coefficient $C$ is a simple by-product of the previous depth estimations; its explicit expressions are derived.

\subsection{The three sub-regimes and the observational term $\mathcal{Q}_{H}$}

Let us define the three sub-regimes, from fully sheared to pure slip, as follows.

- Sub-regime 1 (sr1): the fully-sheared sub-regime which corresponds to a vanishing friction coefficient $(C \approx 0)$; hence the slip ratio $R_{s}$ tends to 0 .

- Sub-regime $2(\mathrm{sr} 2)$ : the mildly-sheared regime in the sense $C \approx A h$. Recall that $R_{s}=0.5$ corresponds to $(q+1) C=2 A h$.

- Sub-regime 3 (sr3): the weakly sheared regime in the sense $C>>A h$, hence the slip ratio $R_{s}$ tends to 1 .

A formal error estimate of the asymptotic xSIA equation presented in [4] demonstrates that sub-regime 2 is the limit case of the xSIA equation validity. In other words for $\mathrm{C}$ larger, the a-priori scaling made to obtain the xSIA equation breaks down. Indeed, this a-priori scaling (roughly $\sigma_{x z}>>\sigma_{x x}$ ) is not valid anymore in the sub-regime 3, see [40] too. Nevertheless, it will be demonstrated that the depth estimate obtained but assuming $C>A h$ (sub-regime 3 case) is a particular case of those obtained in the sub-regime 2 case.

The observational surface term $\mathcal{Q}_{H}$. The surface quantity which naturally appears in the forthcoming expressions is the ratio between the surface velocity norm and the surface slope norm power $q$ :

$$
\mathcal{Q}_{H}=\frac{\left|\mathbf{u}_{H}\right|}{\mathcal{S}^{q}}
$$

Then the slip ratio $R_{s}$ defined by (6) can be read in function of $\mathcal{Q}_{H}$ and the depth $h$ as follows:

$$
R_{s}=\frac{2 \bar{\rho} \underline{\mathrm{A}}}{(q+1)} \frac{h^{q+1}}{\mathcal{Q}_{H}}
$$

\subsection{Sub-regime 1: explicit expression of $h$}

Let us consider a vanishing friction coefficient $C$. It follows the usual SIA expression of $\mathbf{u}_{H}: \mathbf{u}_{H}=\frac{2 \bar{\rho} A}{(q+1)} \mathcal{S}^{q-1} \mathbf{S} h^{q+1}$. Then from the surface measurements $\left(\mathbf{u}_{H}, \mathcal{S}\right)$, the depth $h$ can be straightforwardly estimated by:

$$
h \approx\left[\frac{(q+1)}{2 \bar{\rho} \underline{A}} \mathcal{Q}_{H}\right]^{1 /(q+1)} \equiv h_{s r 1}
$$

Note that this depth estimate does not depend on the vectorial features of the surface measurements; it depends on norms only.Let us recall that sub-regime 1 corresponds to the "traditional SIA regime" and few studies has addressed the depth inversion in this case, see the general introduction. The forthcoming numerical results demonstrate that the present estimation is not accurate as soon as the slip ratio is lower than 0.85 approximatively. 


\subsection{General case, sub-regime 2: polynomial in $h$ and depending on $\eta$}

Sub-regime 2 corresponds to a mildly sheared regime hence much faster flows than sub-regime 1 . Nevertheless, this sub-regime still corresponds to the xSIA model validity, see [4]. The typical case is $R_{\text {slip }} \approx 0.5$ or equivalently $(q+1) C \approx 2 \underline{A} h$.

Polynomial in $h$. Given the observed quantity $\mathcal{Q}_{H}$, using the surface velocity expression (5), it follows: $\left[C+\frac{2}{q+1} \underline{A} h\right] h^{q}=\frac{\mathcal{Q}_{H}}{\bar{\rho}}$. This expression combined with the expression of the effective diffusivity $\eta$, see (7), leads to the following non linear algebraic system in $(h, C)$ :

$$
\left\{\begin{array}{l}
{\left[C+\frac{2}{q+1} \underline{A} h\right] h^{q}=\frac{\mathcal{Q}_{H}}{\bar{\rho}}} \\
{\left[C+\frac{2}{q+2} \bar{A} h\right] h^{q+1}=\eta}
\end{array}\right.
$$

Let us point out that these two equations are not redundant. Indeed, the first equation is based on the momentum equation only while the second equation results from the depth integration of the horizontal velocity profile i.e. the ice flux, then leading to the expression of $\eta$. Also it can be noted that the second equation can be derived by combining the first equation and the free surface dynamics equation.

By injecting the first equation (the observed surface expression) of (11) into the second one (the effective diffusivity expression), it follows the following polynomial in $h$ of degree $(q+2)$,

$$
a_{2} h^{q+2}-\frac{\mathcal{Q}_{H}}{\bar{\rho}} h+\eta=0
$$

with: $a_{2}=m[(q+2) \underline{A}-(q+1) \bar{A}]$ and $m=\frac{2}{(q+1)(q+2)}$. For the standard case $q=3$, the polynomial is order 5 with $m=\frac{1}{10}$.

Given $\eta$, and the observed quantity $\mathcal{Q}_{H}$, the depth $h_{s r 2}$ can be inferred by computing the roots of Polynomial (12). In next section, it is classically done by computing the eigenvalues of the companion matrix. Since considering the two terms in the analytical expressions (the slip term and the deformation term), the resulting depth estimation applies in the three sub-regime cases.

Isothermal case. In the isothermal case, $a_{2}=m A$ and the polynomial reads: $\frac{\mathcal{Q}_{H}}{\bar{\rho}}\left(\frac{R_{s}}{(q+2)}-1\right) h+\eta=0$ with $R_{s}$ satisfying (9). Hence the depth expression in the sub-regime 2 case reads:

$$
h=\left[1-\frac{R_{s}}{(q+2)}\right]^{-1} \frac{\bar{\rho}}{\mathcal{Q}_{H}} \eta \equiv h_{s r 2}
$$

Even if $\eta$ is given, this explicit expression cannot be evaluated in practice since the slip ratio $R_{s}$ is a-priori unknown. Nevertheless, this expression is useful to analyse the depth estimate sensitivity with respect to the variations of $\mathcal{Q}_{H}$ or with respect to $R_{s}$. In particular it is worth to notice that this estimation varies from $\frac{\bar{\rho}}{\mathcal{Q}_{H}} \eta$ to $\frac{(q+2)}{(q+1)} \frac{\bar{\rho}}{\mathcal{Q}_{H}} \eta$ when $R_{s}$ varies from 0 to 1 , therefore not an important variability in terms of $R_{s}$ for $q=3$. Note that this estimation includes the sub-regime 1 one : consider (10) with $R_{s}=1$ and $C=0$.

\subsection{Sub-regime 3: explicit expression of $h(\eta)$}

In the case of sub-regime $3, A h$ is negligible compared to $C$, then the algebraic system (11) simplifies as follows: $C h^{q} \approx \frac{1}{\bar{\rho}} \mathcal{Q}_{H}$ and $C h^{q+1} \approx \eta$. The depth expression follows:

$$
h \approx \frac{\bar{\rho}}{\mathcal{Q}_{H}} \eta \equiv h_{s r 3}
$$

Given $\mathcal{Q}_{H}$ and the diffusivity $\eta, h_{s r 3}$ is straightforwardly obtained. Note that the estimation (13) gives (14) by making vanish the slip ratio $R_{s}$.

In next section, it will be presented how the diffusivity $\eta$ can be approximated by Variational Data Assimilation (VDA).

Comparison of the three estimations in the isothermal case. Let us calculate the difference between the two extreme estimations (10) and (14). For the sake of simplicity, the flow is supposed to be isothermal $(\bar{A}=\underline{A}=A)$. From (10), it follows: $\frac{\mathcal{Q}_{H}}{\bar{\rho}}=\frac{2 A}{(q+1)} h_{s r 1}^{q+1}$. While from (14), it follows: $\frac{\mathcal{Q}_{H}}{\bar{\rho}}=\frac{\eta}{h_{s r 3}}$.

If setting $C=0$ in $\eta$ in the sub-regime 3 depth estimation (14), it follows: $\frac{2 A}{(q+1)} h_{s r 1}^{q+1}=\frac{1}{h_{s r 3}} \cdot \frac{2 A}{(q+2)} h_{s r 1}^{q+2}$. Hence: $h_{s r 3}=\frac{(q+1)}{(q+2)} h_{s r 1}$.

In others words, given the $\eta$ value in the fully sheared areas $\left(R_{s}\right.$ close to 1$)$, the depth formula corresponding to the vanishing slip ratio gives a value $\frac{(q+1)}{(q+2)}$ smaller than the true one (hence $20 \%$ smaller in the case $q=3$ ) . Nevertheless, it will be noticed in the forthcoming test cases that the range of value of $\eta$ is extremely large (few orders of magnitudes) hence leading to differences of depth much greater than the present $20 \%$. Nevertheless, in practice this remark is useful to select the correct computed polynomial root of (12). 


\subsection{The resulting friction coefficient $C$}

Given the depth value $h$, whatever if it is the sub-regime 1, 2 or 3 which is considered, the corresponding friction coefficient $C$ can be deduced from (11). Its value can be deduced equivalently from the first equation or from the second equation, hence:

$$
C\left(\mathcal{Q}_{H} ; h\right)=\frac{1}{\bar{\rho} h^{q}} \mathcal{Q}_{H}-\frac{2}{(q+1)} \underline{A} h \text { or } C(\eta, h)=\frac{\eta}{h^{(q+1)}}-\frac{2}{q+2} \bar{A} h
$$

Let us point out that despite the observed surface term is uncertain and despite the VDA process to compute $\eta$ is not exact too, in practice both expressions give extremely close numerical values of $C$.

\subsection{Identification of the effective diffusivity $\eta$ by Variational Data Assimilation}

In sub-regimes 2 and 3, the depth estimations depend on the diffusivity $\eta$ defined by (7). We present below how to compute $\eta$ by solving a linear-quadratic optimal control problem.

\subsubsection{Set up of the VDA problem}

Let us recall that the direct model reads as follows, see $(7):-\bar{\rho} \operatorname{div}_{x y}\left(\eta(C, h) \mathcal{S}^{q-1} \nabla H\right)=\dot{a}$, plus boundary conditions. It is a linear elliptic equation in $H$. Then the typical observation function is defined by: $J(\eta ; H)=$ $\frac{\alpha_{H}}{2} \int_{\Omega}\left(H^{\eta}-H^{o b s}\right)^{2} d x+\frac{\alpha_{\eta}}{2} \int_{\Omega}|\nabla \eta|^{2} d x$. And the cost function reads: $\bar{j}(\eta)=J\left(\eta ; H^{\eta}\right)$ where $H^{\eta}$ is the unique solution of the linear equation (7), given $\eta$

Then, given the surface measurements elevation - slope $(H, \mathcal{S})$, the spatially distributed coefficient $\eta$ can be inferred by solving the optimal control problem: $\min _{\eta} j(\eta)$. Since this optimal control problem is linear quadratic then it admits a unique optimal solution $\eta^{*}$.

In practice, it turns out that the quantity $\eta$ varies greatly ( $\approx 4$ orders of magnitude in a multi-regime flow),

then a log change of variable is introduced; the control variable becomes $\omega=\ln (\eta)$. In other respect, it has been observed that considering the $L^{4}$ norm instead of the $L^{2}$ norm in the regularizing term leads to better results. Then the cost function to be minimized is defined as follows:

$$
j(\omega)=\alpha_{H} j_{H}(\omega)+\alpha_{r e g} j_{r e g}(\nabla \omega) \text { with } j_{H}(\omega)=\frac{1}{2} \int_{\Omega}\left|H^{\omega}-H^{o b s}\right|^{2} d x \text { and } j_{r e g}(\nabla \omega)=\frac{1}{2} \int_{\Omega}|\nabla \omega|^{4} d x
$$

with $\left(\alpha_{H}, \alpha_{r e g}\right)$ to be set. The optimal control problem reads:

$$
\min _{\omega} j(\omega)
$$

Let us point out that (17) is no longer a linear-quadratic optimal control problem (the cost function is not quadratic anymore due to the log change of variables) but the cost function remains strictly convex and the inverse problem still admits a unique optimal solution $\omega^{*}$.

Remark 1. The airbone or in-situ measurements can been used for the definition of the first depth estimation $h^{(0)}$. Moreover they can be directly assimilated as follows. From the depth mesurements $h^{\text {obs }}$, the friction coefficient can be deduced from (15) next the effective diffiusivity $\eta$ from (7) hence the value $\omega^{\text {obs }}$. Then the following quadratic misfit term is added to the (total) cost function $j: j_{\omega}(\omega)=\frac{1}{2} \int_{\Omega}\left|\omega-\omega^{o b s}\right|^{2} d x$.

Let us point out that in the numerical results presented in this study, this term $j_{\omega}$ has not been considered. For real test cases (hence with many uncertainties sources) it is obvious that all available depth measurements should be assimilated.

Remark 2. On the impossibility to directly assimilate $\mathbf{u}_{H}$ into the $x S I A$ equation. The misfit between the xSIA model and the surface observations can be based on the elevation measurements $H$ only (e.g. acquired by altimetry) and not on the surface velocities $\mathbf{u}_{H}$ since the latter depends on the unknown pair $(h, C)$, see $(5)$. Thus the observations $\mathbf{u}_{H}$ (e.g. derived from InSAR) are not directly assimilated by the present VDA process. However $\mathbf{u}_{H}$ appears in the three depth estimations through the observational term $\mathcal{Q}_{H}$, see $(10)(13)(14)$.

\subsubsection{The equations and numerical methods}

The direct model (7) is numerically solved using a standard Lagrange finite element method, by employing the Fenics Python library [1,23]. The weak formulation consists to find $H \in V_{t}$ such that: $\forall \phi \in V_{0}, a(\omega ; H, \phi)=b(\phi)$ with

$$
a(\omega ; H, \phi)=\bar{\rho} \int_{\Omega} \mathcal{S}^{q-1} e^{\omega} \nabla H \nabla \phi d x \text { and } b(\phi)=\int_{\Omega} \dot{a} \phi d x
$$


and $V_{0}=W^{1, q^{\prime}}(\Omega)$ an adequate Banach space $\left(q^{\prime}\right.$ depending on $\left.q, q \geq 1\right), V_{t}$ its corresponding affine sub-space (taking into account the non-homogeneous Dirichlet boundary condition).

For $q=3$ and under an assumption on $\eta=e^{\omega}$ (it has to remain bounded), the existence and uniqueness of the weak solution $H \in V_{t}$ can be addressed using mathematical analysis tools of non-linear elliptic problems (variational methods and/or fixed point theorems), see e.g. [8, 9]. The detailed mathematical and finite element analysis of this equation (with extra assumptions) can be found in [20, 19].

Next, the cost function (16) can be computed. The optimal control problem (17) is numerically solved by Variational Data Assimilation (VDA); it is based on the adjoint equations and the gradient of the cost function (first order optimization method), see e.g. [28] for details.

The adjoint equations read as follows. Given $\omega$, given $H^{\omega}$ the unique solution of the state equation / direct model (18), find $p^{\omega} \in V_{0}$ such that: $\partial_{H} a\left(\omega ; H^{\omega}, p\right) . z=\partial_{H} J\left(\omega ; H^{\omega}\right) . z$ for all $z \in V_{0}$.

Since the state equation is linear symmetric, it follows: $\partial_{H} a(\omega ; H, p) . z=a(\omega ; p, z)$.

Also: $\partial_{H} J\left(\omega ; H^{\omega}\right) . z=\int_{\Omega} z\left(H^{\omega}-H^{o b s}\right) d x$.

The adjoint equation can be solved by the same finite element method as the state equation.

Given the (unique) adjoint $p^{\omega}$, the gradient of the cost function reads, see e.g. [28] Chapter 3:

$j^{\prime}(\omega) \cdot \delta \omega=\partial_{\omega} J\left(\omega ; H^{\omega}\right) . \delta \omega-\left[\partial_{\omega} a\left(\omega ; H, p^{\omega}\right) . \delta \omega-\partial_{\omega} b\left(\omega ; p^{\omega}\right) . \delta \omega\right] \forall \delta \omega$, which gives:

$$
j^{\prime}(\omega) \cdot \delta \omega=2 \alpha_{r e g} \int_{\Omega}|\nabla \omega|^{2} \nabla \omega \nabla(\delta \omega) d x-\bar{\rho} \int_{\Omega} \mathcal{S}^{q-1} e^{\omega} \delta \omega \nabla H^{\omega} \nabla p^{\omega} d x \quad \forall \delta \omega
$$

After discretization (control variable included), this gives the gradient: $\left\langle\nabla j, \delta \omega_{h}\right\rangle=j^{\prime}\left(\omega_{h}\right) \cdot \delta \omega_{h}$, where the index $h$ denotes the finite element variable. The employed minimization algorithm is the L-BFGS algorithm (quasi-Newton method, Python scipy procedure). The complete computational software has been fully assessed by implementing rigorous tests (explicit solutions with convergence curves); also the gradient computations have been compared to convergent finite difference values, see e.g. [28] Chapter 6 for details.

\subsubsection{The complete VDA process}

In all the sequel the complete VDA process is performed in two stages:

- A first one (VDA\#1) by making fit the model output with the measured surface elevations only, i.e. $\alpha_{\text {reg }}=0$ in $(16)$.

- A second one (VDA\#2), starting from the VDA\#1 solution and by minimizing (16) with $\alpha_{\text {reg }}$ empirically tuned "at best". The goal is to make a balance between the data misfit and the regularized solution (in particular in presence of noise in data).

Twin experiments. In the present study, only synthetic data are considered, then the VDA experiments are twin experiments. This consists to first generate the data by running the direct model. Next, a Gaussian noise is added or not, depending on the experiment. Then the goal is to infer the unknown parameters by the inverse method and compare the computed quantities with the original - true ones. An important point is define a good first guess $\eta^{(0)}$; it is directly obtained from the a-priori slip ratio law $R_{s}^{(0)}$ and the first depth estimation $h^{(0)}$ obtained previously.

Stopping criteria. Few classical criteria has to be evaluated before deciding the minimization algorithm has converged: the stationarity of $j$ and $\omega$, the gradient value $\nabla j$ in particular. In practice, the present minimization processes have been stopped when entering in the "over-fitting zone" i.e. when the model misfits become smaller than the measurement uncertainties. Here the virtual surface elevation $H^{\text {obs }}$ are supposed to be accurate at $+/-10 \mathrm{~cm}$. This convergence criteria has always been reached in approximatively 30 iterations.

Orders of magnitude and accuracy. The unknown effective diffusivity $\eta$ presents few orders of magnitude (hence this log change of variables) but also with extremely stiff local variations. This features make the inverse problem particularly challenging in terms of numerics.

Thus the obtained numerical accuracy (on the optimal diffusivity $\eta^{*}$ ) is not as accurate as it can be expected in a standard linear-quadratic case. Typically, for a standard linear-quadratic VDA problem (with more gentle control variable variations) a few $\%$ error can be easily reached; on the contrary, in presence of the large and stiff variations of the optimal control variable, a dozen of \% error only is reached, see the numerical results in next section.

\section{The multi-regime test cases}

In this section, the multi-regime test case aiming at assessing the inverse method above is described. The considered multi-regime flows include many features of the real world flows in terms of fluid mechanics (i.e. independently of the measurement difficulties and uncertainties). Following $[\mathbf{3 , 1 2}, \mathbf{2 5}]$, the bed topography is 


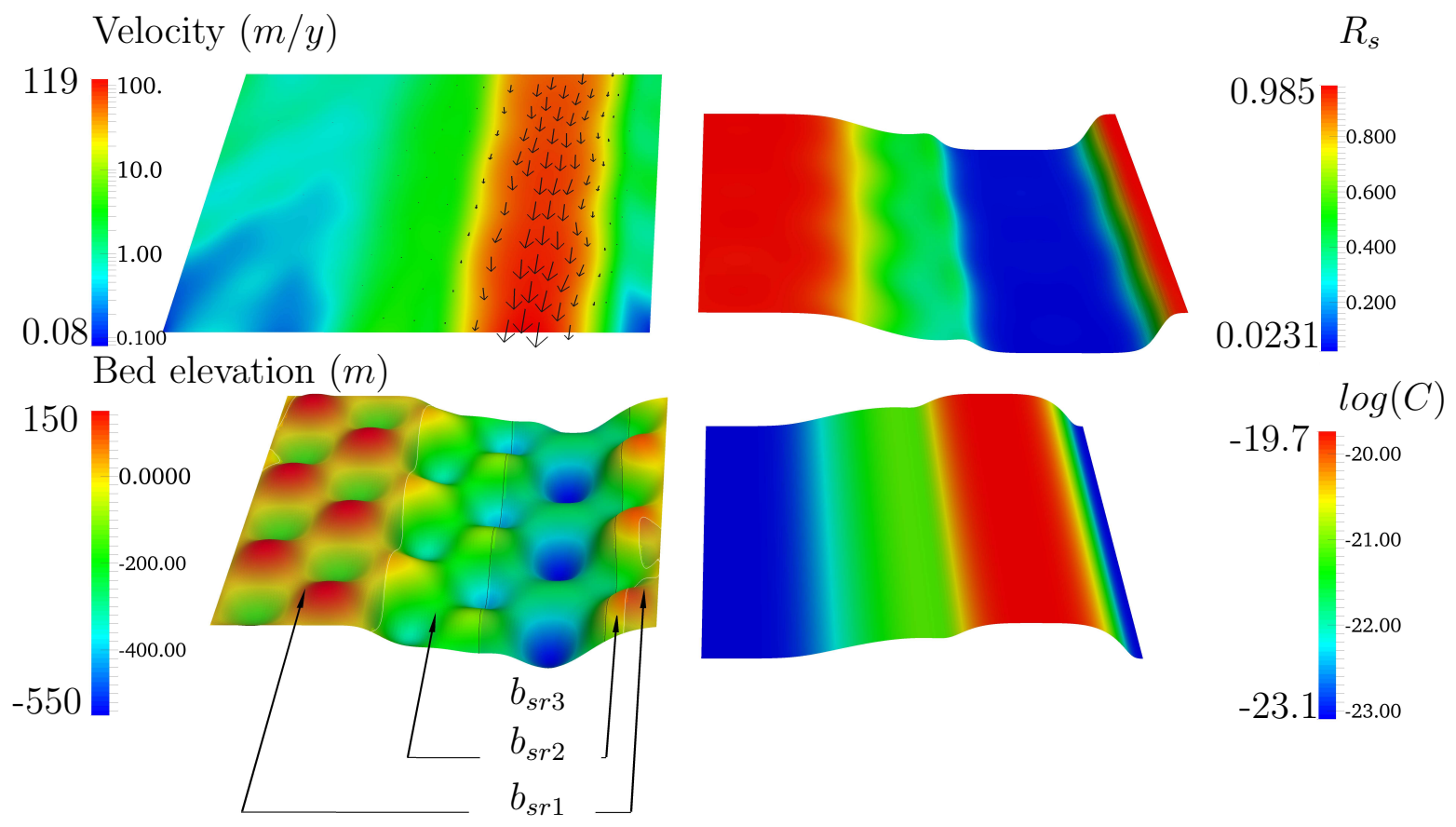

Figure 2: The multi-regime test case. (Up) Left: surface velocity $\mathbf{u}_{H}$ (in m/y). Right: slip ratio $R_{s}$. (Down) Left: bed elevation $b$. (in $\mathrm{m}$ ) Right: friction coefficient $C$ (in $\log$ scale). White lines on the bed elevation denote the sub-regime boundaries defined by Table 1 .

defined consistently with the low-band filter feature of these shallow flows; in particular it respects the minimal wave lengths measurable from the surface. The sub-regime areas can be defined quite accurately from the surface velocity values only. Next two measurement scenarios are defined: depth measurements are available downstream the flow (it is the case 1) or laterally, approximatively parallel to a flow line and within a very slow flow area only (it is the case 2). Both cases define difficult inverse problems which have not been solved up to now in the literature (for the reasons detailed in the last paragraph).

\subsection{Flow description}

The geometrical domain $\Omega$ is a square of length $L=100 \mathrm{~km}$. The surface elevation $H$, the slopes $\mathcal{S}$, and the bed elevation $b$ are presented in Fig. 3. The flow presents the whole range of slip ratio $R_{s}$ : from 0.02 to 0.98 , see Fig. 2; hence this flow contains the three sub-regimes.

The bed presents sinusoidal variations which are consistent with the mesh size $(\approx 10$ points minimum per wave length) and consistent with the depth $(\approx 5$ depth values minimum per wave length). For more details on the shallow ice flow filtering features, the reader can refer to the detailed and complementary analysis presented in $[3,12,25]$.

The test case definition is a crucial point to assess in a reliable way the inference method. It has to represent a real like flow (hence multi-regime) and it has to be consistent with the shallow ice flow - xSIA model filtering features. The flow is assumed to be isothermal with $q=3$ and $A=3 \cdot 10^{-24}$ (this value corresponds to ices at $\approx-5^{\circ} C$ ); also: $\rho=934$ and $g=9.81$. The mass balance term $\dot{a}=0$.

The bed elevation is defined by: $b(x, y)=150 \sin \left(\frac{6 \pi}{L} x\right) \sin \left(\frac{6 \pi}{L} y\right)+400 e^{-\frac{\left(y-\frac{3}{4} L\right)^{2}}{2 \times 9 e 07}}+200 e^{-\left(\frac{(y-L / 2)^{2}}{2 \times 9 e 07}\right)^{2}}$

The friction coefficient is defined by: $C(x, y)=\frac{10^{3} A}{(q+2)} \frac{1}{50}\left[1+50^{2} \exp \left(-\frac{(y-L / 2)^{2}}{2 \times 9 e 07}\right)+50 \exp \left(-\frac{(y-L / 2)^{4}}{(2 \times 9 e 07)^{2}}\right)\right]$.

The definitions of $b$ and $C$ above generate a canyon draining a faster ice stream, see Fig. 2. The surface elevation $H$ is obtained by solving the xSIA equation (7) with Dirichlet boundary conditions. The latter read: $H(x, y)=1000-5 \cdot 10^{-3} x$ for all $x \in \partial \Omega$. The lateral boundary surfaces present a longitudinal mean slope equal to $5 \%$. The computational finite element mesh is a structured triangular mesh $140 \mathrm{x} 140$ with refinements in the sharpest bed variation areas, in particular in the vicinity of the left boundary of the canyon (in the flow direction). The resulting surface elevation $H$ and slope values $\mathcal{S}$ are plotted in Fig. 3. The surface slopes are computed for each mesh triangle; they range from $0.3 \%$ to $0.8 \%$.

It can be noticed that the bed bumps have a clear effect on the top surface slopes. On the contrary, the 


\begin{tabular}{|c|c|c|}
\hline Sub-regime & Velocity norm $\left|\mathbf{u}_{H}\right|(m / y)$ & Corresponding slip ratio $R_{s}$ \\
\hline sr1: fully sheared & $\left|\mathbf{u}_{H}\right| \leq 1$ & $R_{s} \gtrsim 0.8$ \\
\hline sr2: intermediate & $1 \leq\left|\mathbf{u}_{H}\right| \leq 10$ & $0.1 \lesssim R_{s} \lesssim 0.8$ \\
\hline sr3: full sliding & $\left|\mathbf{u}_{H}\right| \geq 10$ & $R_{s} \lesssim 0.1$ \\
\hline
\end{tabular}

Table 1: Sub-regime area defined from the surface velocity values.

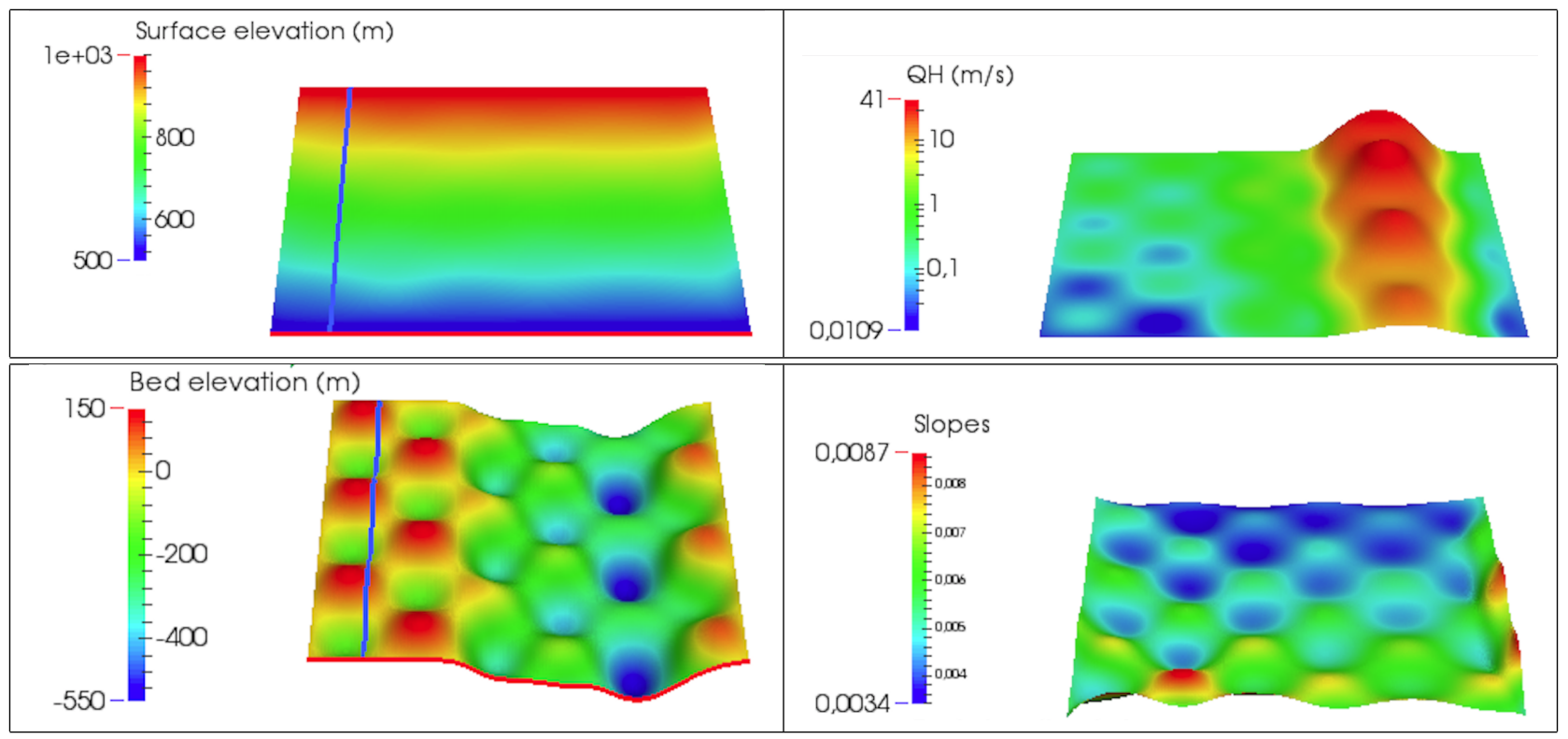

Figure 3: The multi-regime test case. (Left) Up: surface elevation $H$ (in m). Down: bed elevation $b$ (in $\mathrm{m}$ ). The lateral blue line and the downstream red line (figures up and down) denote the depth measurement locations (e.g. flight tracks).

(Right) Up: the observational term $\mathcal{Q}_{H}=\frac{\left|\mathbf{u}_{H}\right|}{\mathcal{S}^{q}}$ (in $\log$ scale). Down: the slope values $\mathcal{S}$.

canyon location cannot be visually detected neither from the surface elevation nor from the surface slopes, see Fig. 3.

Let us point that the sinusoidal bed variations respect the minimal wavelengths not fully filtered by the (shallow) flow, see $[\mathbf{3}, \mathbf{1 2}, \mathbf{2 5}]$. Also the minimal grid points per wave length is respected.

The regime transitions are mainly due to the friction coefficient variations, see Fig. 2, and these variations are extremely stiff: few orders of magnitudes within a narrow space region (hence almost discontinuities with 1 or 2 order of magnitude gaps). Then an accurate inference of $C$ is obviously very difficult. The numerical results below demonstrate that the present inverse method makes possible an accurate depth inference despite the large and stiff variations of $C$.

\subsection{Sub-regime areas definition}

The sub-regime areas corresponds to the balance between the deformation term in $A h$ vs the sliding term in $C$,

see (5). The three different situations (sub-regimes 1, 2 and 3) correspond roughly to the slip ratio range values indicated in Table 1. It turns out that each sub-regime area can be easily defined from the surface velocity norm values $\left|\mathbf{u}_{H}\right|$.

The resulting sub-regimes boundaries are plotted in white and black on the bed elevation maps, see e.g. Fig. 2. The white lines denote the sub-regime 1 boundaries, the black lines denote the sub-regime 3 boundaries; hence the sub-regime 2 area is defined by the white and black lines.

On the few orders of magnitude of $\eta$. The surface velocity presents 3 orders of magnitude (from 0.1 to $100 \mathrm{~m} / \mathrm{y}$ ); this leads to a friction coefficient $C$ presenting 4 orders of magnitude, see Fig. 2. Such a large variation range is common in ice models. Then the observational quantity $\mathcal{Q}_{H}$ defined by (8) presents $3-4$ orders of magnitude too, see Fig. 3. The effective diffusivity $\eta$ defined by (7) presents patterns very similar to $\mathcal{Q}_{H}$ ones, with 4 orders of magnitude too.In the sequel, the effective diffusivity $\eta$ will be computed by a VDA process; then its large and locally stiff variations makes its numerical inference quite unaccurate in the stiffer variation areas. 
Let us notice that these large variations of $\eta$ are due to the depth variations and not to the regime (or equivalently slip ratio) variations. Indeed, in the sub-regime 1 case, if setting $C=0, R_{s l i p}=1$, then: $\eta_{s r 1}=$

$\frac{2 A}{(q+2)} h^{q+2}$. Hence $\eta$ varies with few orders of magnitude since $\eta \sim h^{q+2}$. Also, in the case of sub-regimes 2 and $3, \eta=\eta_{s r 1}+C h^{q+1}$. Typically for $R_{s}=0.5,(q+1) C=2 A h$ and: $\eta=\left[\frac{(2 q+3)}{(q+1)(q+2)}\right] 2 A h^{q+2}=\frac{(2 q+3)}{(q+1)} \eta_{s r 1}$. In the case $q=3$, it gives: $\eta=\frac{9}{4} \eta_{s r 1}$. Therefore the large variations of $\eta$ are not due to the regime variations but to the depth variations.

\subsection{Two scenarios of measurements: downstream (Case 1) and lateral (Case 2)}

In the following the depth $h$ is inferred following the method described previously, next the friction coefficient $C$ is inferred. Two scenarios depending on the measurement locations are considered:

- Case 1: the depth is measured downstream the flow, see the red line in Fig. 3.

- Case 2: the depth is measured laterally only, following the blue line in Fig. 3.

Both cases are difficult inverse problems for few reasons. In Case 1, the measurement is available downstream and not upstream. The configuration in Case 2 is an even lmore challenging since the measurements are more or less parellel to a single stream line hence any method based on an hyperbolic equation (like the depth mass equation, even if regularized by artificial diffusion) can infer a reliable depth since the given information is not cross-stream. Moreover the measurements "miss" the canyon hence "miss" the large bed variations. Then no reference relationship between the surface velocity and the bed variations can be stated from the measurements. The present inverse method remains robust independently of the measurement location(s) since based on an elliptic equation.

\section{The first depth estimation $h^{(0)}$}

Given the surface observations (elevation and velocities), given the depth measurements at few points (e.g. a flight track over an ice-shed in Antarctica or Greenland), an a-priori slip ratio law $R_{s}^{(0)}$ can be derived. From this a-priori law $R_{s}^{(0)}$, using the few depth estimations derived previously (Section 3), a first estimation is obtained. This estimation denoted by $h^{(0)}$ turns out to be already excellent. Next this value $h^{(0)}$ is improved by the VDA process $\left(h^{(0)}\right.$ is the first guess value).

\subsection{From the depth measurements to the a-priori slip ratio law $R_{s}^{(0)}$}

As already mentionned, the depth measurements along the lines/tracks indicated in Fig. 3 combined with the surface measurements $\left(H,\left|\mathbf{u}_{H}\right|\right)$ make possible to obtain a local slip ratio law since $R_{s}$ can be written as a function of $h$ and $\mathcal{Q}_{H}$, see (9). Recall $q$ and $\underline{\mathrm{A}}$ are given. Fig. 4 represents the slip ratio $R_{s}$ vs the surface velocity $\left|\mathbf{u}_{H}\right|$; the blue dots are the measured values. These measurements are plotted for the two scenarios, Case 1 and Case 2, corresponding to Fig. 4) (Left) and (Right) respectively. Next, given the measured slip ratio $\operatorname{graph}(\mathrm{s})$ (the blue points in Fig. 4), a least-square approximation gives an a-priori slip ratio law $R_{s}^{(0)}$ : the red dots in both figures.

In Case 2, the measured slip ratio range is extremely small hence making the derivation of an a-priori slip ratio law difficult. Then two extra empirical values are added to the measurement values: $\left(\mathbf{u}_{H}=1 \mathrm{~m} / \mathrm{y}\right.$, $R s=0.5)$ and $\left(\mathbf{u}_{H}=20 \mathrm{~m} / \mathrm{y}, R_{s}=0.03\right)$. These two extra values are the two isolated points indicated in green in Fig. 4 (Right).

The complete relation $R_{s}\left(\mathcal{Q}_{H}, h\right)$ for all points of the computational domain (i.e. including the non measured values) are represented by the blue crosses.

The slip ratio law $R_{s}$ can be plotted vs the (complete) observational term $\mathcal{Q}_{H}$ (not shown here). However since the variation range of $\mathcal{Q}_{H}$ is much larger than those of $\mathbf{u}_{H}$, it is more difficult to infer a good a-priori law $R_{s}^{(0)}$. A comparison between two resulting a-priori slip ratio laws (one obtained from $\left|\mathbf{u}_{H}\right|$ measurements, the other from $\mathcal{Q}_{H}$ measurements) is presented in Fig. 5. As expected, the velocity-derived law is closer to the true slip ratio map. The $\mathcal{Q}_{H}$-derived law makes appear the bed bumps since the presence of $\mathcal{S}^{-q}$ in the definition of $\mathcal{Q}_{H}$, see Fig. 5 .

\subsection{The first depth estimation $h^{(0)} \equiv h\left(\mathcal{Q}_{H}, R_{s}^{(0)}\right)$}

Recall that given the observational surface term $\mathcal{Q}_{H}$, given the slip ratio values $R_{s}$, the depth expression reads in all sub-regimes as follows: 

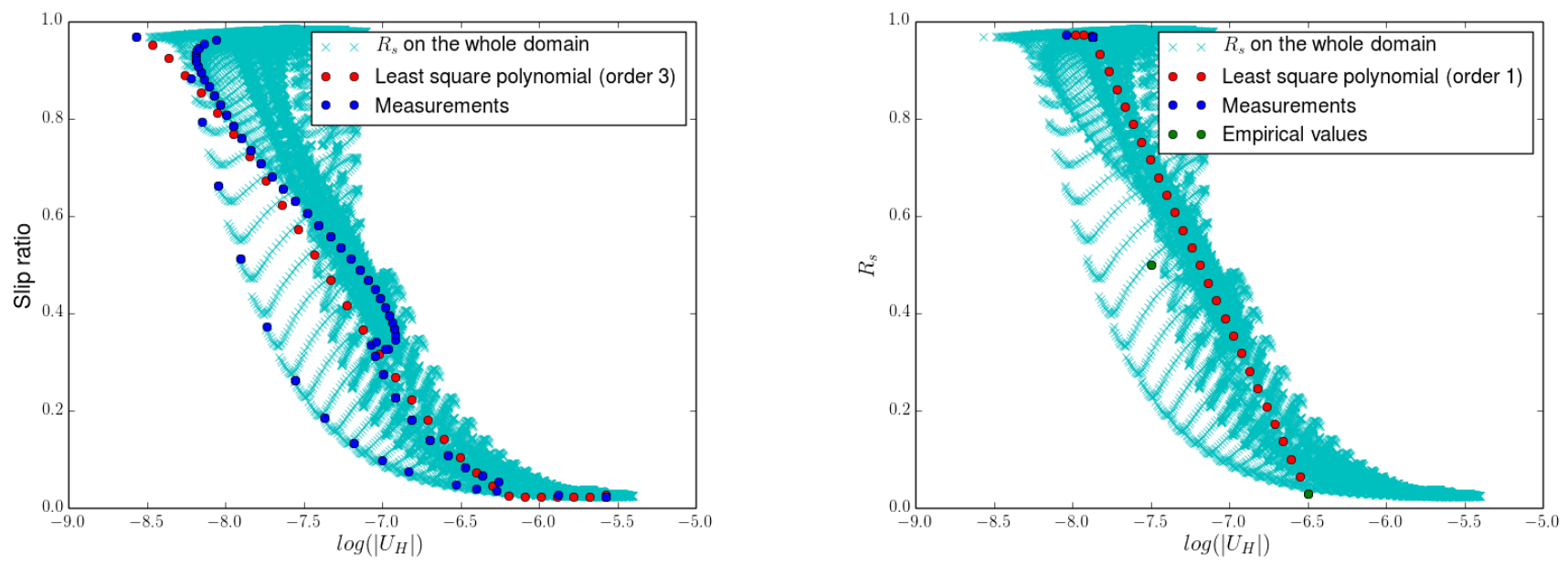

Figure 4: Slip ratio vs $\left|\mathbf{u}_{H}\right|$ (in $\log$ scale) for test case 1 (downstream measurements) (Left) and test case 2 (lateral measurements) (Right). The blue dots are the measurements. The cyan crosses are the values throughout the domain (they are not measured). The red dots are the resulting a-priori slip ratio law, obtained by least-squares.

\section{$R_{s}$}

\begin{tabular}{|lrl|l|l|l}
0.200 & 0.400 & 0.600 & 0.800 & \\
\hline 0.0231 & & & 0.985
\end{tabular}

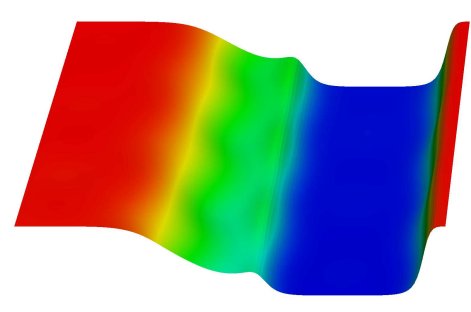

True

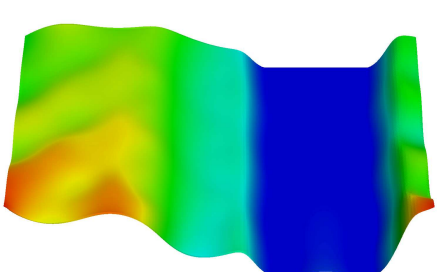

Velocity based

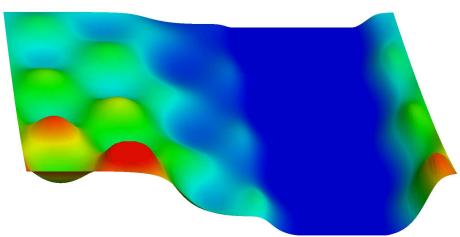

$\mathcal{Q}_{H}$ based

Figure 5: Slip ratio values: True values $R_{S}$ (Left). A-priori law $R_{s}^{(0)}$ derived from: $\left|\mathbf{u}_{H}\right|$ only (Middle); from $\mathcal{Q}_{H}=\frac{\left|\mathbf{u}_{H}\right|}{\mathcal{S}^{q}}$ (Right). 


$$
h^{(0)} \equiv h\left(\mathcal{Q}_{H}, R_{s}\right)=\left[\frac{(q+1)}{2 \bar{\rho} \underline{A}} \mathcal{Q}_{H} R_{s}\right]^{\frac{1}{(q+1)}}
$$

Given the a-priori slip ratio law $R_{s}^{(0)}$, this relation provides directly the first depth estimation $h^{(0)}$. Note that: $h^{(0)}=h\left(\mathcal{Q}_{H}, R_{s}^{(0)}\right) \sim\left[\mathcal{Q}_{H} R_{s}\right]^{\frac{1}{(q+1)}}$; then the accuracy of $h^{(0)}$ depends directly on the accuracy $R_{s}^{(0)}$ (power-law in $\left.\frac{1}{(q+1)}\right)$.

The first estimation (19) with $R_{s}=1$ gives the estimation $h_{s r 1}$, see (10). However for high values of $R_{s}$ (sub-regime 1 areas), $h$ directly calculated from (10) is more accurate than if evaluating (19). This is due to the quite low accuracy of $R_{s}^{(0)}$ for high values, see Fig. 5.

The resulting first guess $\eta^{(0)}$ The first depth estimation $h^{(0)}$ defined by (19) can be improved by performing the VDA process presented previously. This VDA process aims at computing an optimal effective diffusivity $\eta$ from a first guess $\eta^{(0)}$. The latter is straighforwardly calculated from the polynomial expression (12) and $h^{(0)}$ defined above.

\section{Case 1: downstream measurements}

In this section, the numerical results obtained in the case of downstream measurements (the red track in Fig. 3) are presented and analysed. First, the inference process is performed from the exact data. Second, it is performed from perturbed data (the exact surface measurement $\mathcal{Q}_{H}$ plus a random noise).

\subsection{Exact measurements}

Given the downstream depth measurements, see Fig. 3, given the exact surface data $(H, \mathcal{S})$, the VDA process (7)-(17) is performed in two steps:

- VDA\#1: the cost function contains the misfit term only i.e. $\alpha_{\eta}=0$ in (16).

- VDA\#2: the regularization term is added to the cost function, see (16). The goal of this second VDA process is to obtain a reasonably smooth solution while fitting the data.

After each VDA process, the two depth estimations (10) and (14) are explicitly obtained while the sub-regime 2 depth estimation $h_{s r 2}$ is obtained by solving numerically the polynomial (12). The polynomial roots are obtained by computing the eigenvalues of the corresponding companion matrix (Python procedure numpy.roots). The selected root value is those belonging to the interval $\left[h_{s r 3}, h_{s r 1}\right]$ (if imaginary, the real part only is considered).

\subsubsection{VDA\#1: fitting data only}

The VDA problem (7)-(17) is numerically solved with $\alpha_{r e g}=0$. As detailed previously, an a-priori slip ratio map $R_{s}^{(0)}$ is defined, next the first depth estimation $h^{(0)}$ is calculated in the three sub-regime areas, next the first guess $\eta^{(0)}$. It can be noticed that the first depth estimation $h^{(0)}$ is already an excellent estimation of the true depth, see Fig. 6.

Next VDA\#1 process is performed. The minimization process is robust (with respect to the first value) and converge accurately in 30 iterations approximatively (the misfit term is divided by a factor $10^{4}-10^{5}$ ). From the computed optimal value $\eta^{*}$, the depth estimations in sub-regimes 2 and 3 areas are computed; they are plotted in Fig. 7. The three estimations are quite accurate in their respective domain of validity. Typically $h_{s r 1}$ is accurate to within $5 \%$ (and less) in the sub-regime 1 area; $h_{s r 2}$ (resp. $h_{s r 3}$ ) is accurate to within $10 \%$ in the inner part of the sub-regime 2 area (resp. sub-regime 3 area) and accurate to within $20 \%$ at the area boundaries.

The large variations and stiff variations of $\eta$ between the different sub-regime areas make the numerical inverse problem difficult. The numerical inferred value $\eta$ is relatively less accurate, hence the less accurate depth estimation in these areas. Concretely, it gives the local "peaks" of errors $(\approx 25 \%$ error) plotted in Fig. 7 .

Let us recall that by construction the sub-regime2 depth derivation (12) is valid everywhere; however it is not necessarily the most accurate estimation, Fig. 7 , since the respective simplifications made to obtain $h_{s r 1}$ and $h_{s r 3}$ circumvent the errors made on the a-priori slip ratio law $R_{s}^{(0)}$. This is particularly true in the sub-regime 1 case, see Fig. 7.

Following the a-priori definitions of the sub-regime areas, see Table 1, the combination of the 3 estimations $h_{s r} \square$ provide the global bed elevation value. At the sub-regime limits, corresponding to the large and stiff variations of $\eta$, the relative error on $b$ equals approximatively $20 \%$ (corresponding to the "linear peaks" plotted in Fig. 7). Out of these stiff regime transition areas, the relative error is smaller than $10 \%$. 


\begin{tabular}{|c|c|c|c|c|c|c|c|c|}
\hline \multicolumn{4}{|c|}{$R_{s}$} & \multicolumn{2}{|c|}{ Bed } & \multicolumn{3}{|c|}{$\eta$} \\
\hline 0.2 & 0.4 & 0.6 & 0.8 & -400 & 0.00 & $\begin{array}{lll}1 . e-11 & 1 . e-10\end{array}$ & 1.e.-09 & 1.e. 08 \\
\hline 0.02 & & & 0.985 & -550 & 150 & $5.14 \mathrm{e}-12$ & & $8.3 \mathrm{e}-08$ \\
\hline
\end{tabular}
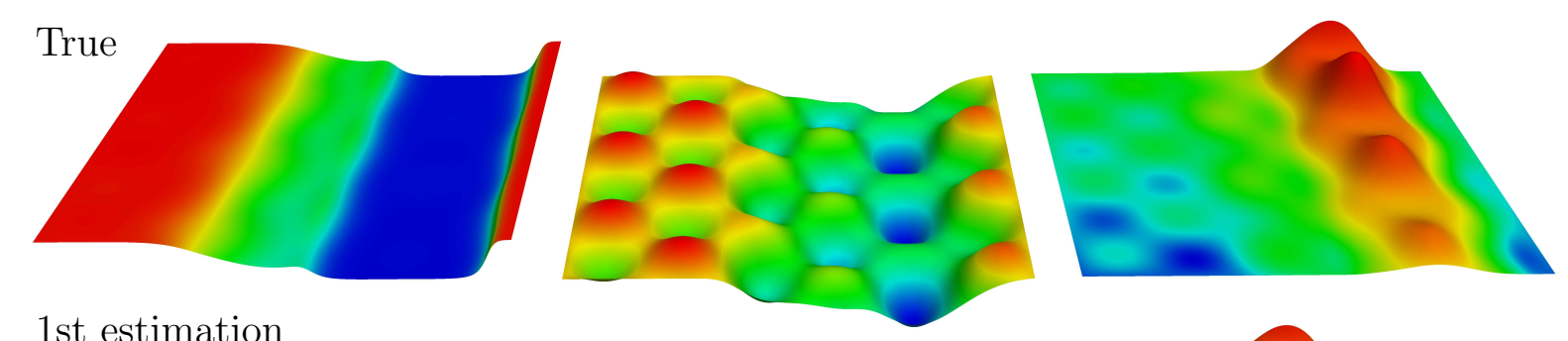

\section{1st estimation}
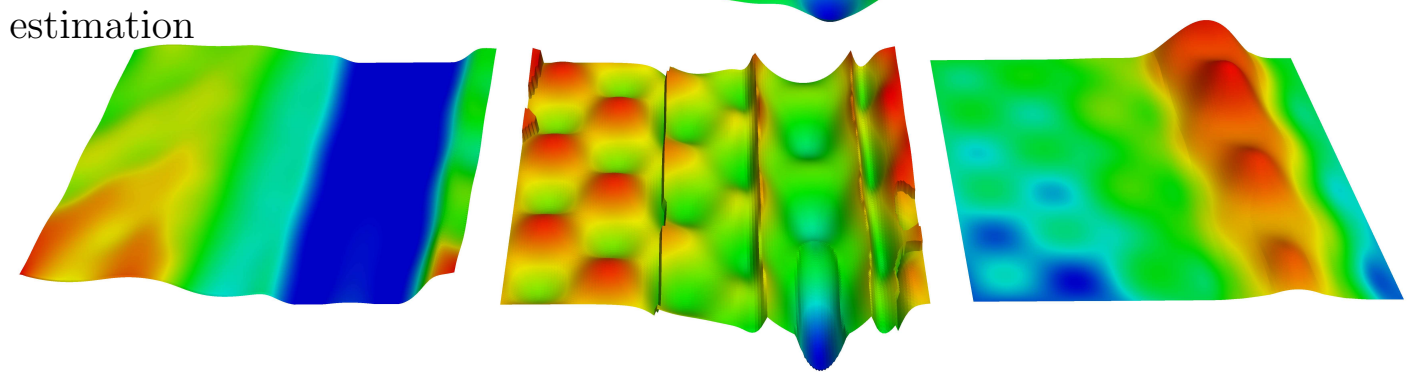

Figure 6: Test case 1 with exact measurements. From left to right : slip ratio $R_{s}$, bed elevation $b$ (in $\mathrm{m}$ ), effective diffusivity $\eta$ (log scale). (Top) True values. (Down) a-priori slip ratio $R_{S}^{(0)}$, 1 st bed estimation $b^{(0)}$ infered from (19), resulting value $\eta^{(0)}$ from (12).

$h$ error (\%)
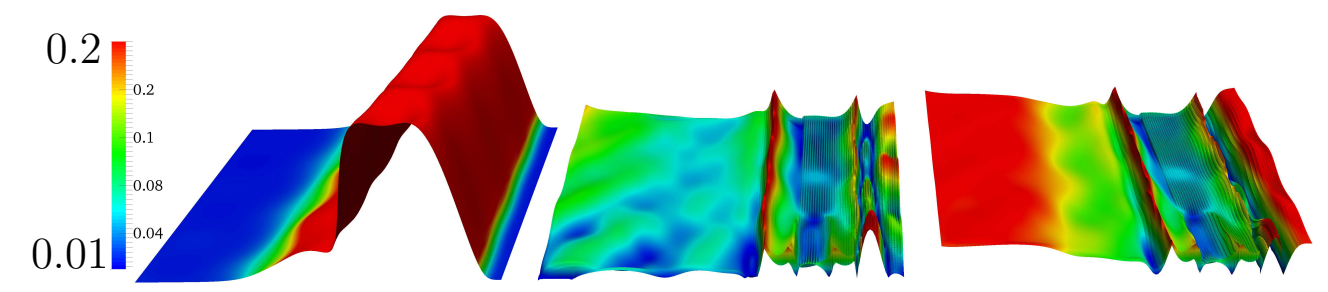

Bed elevation $(m)$
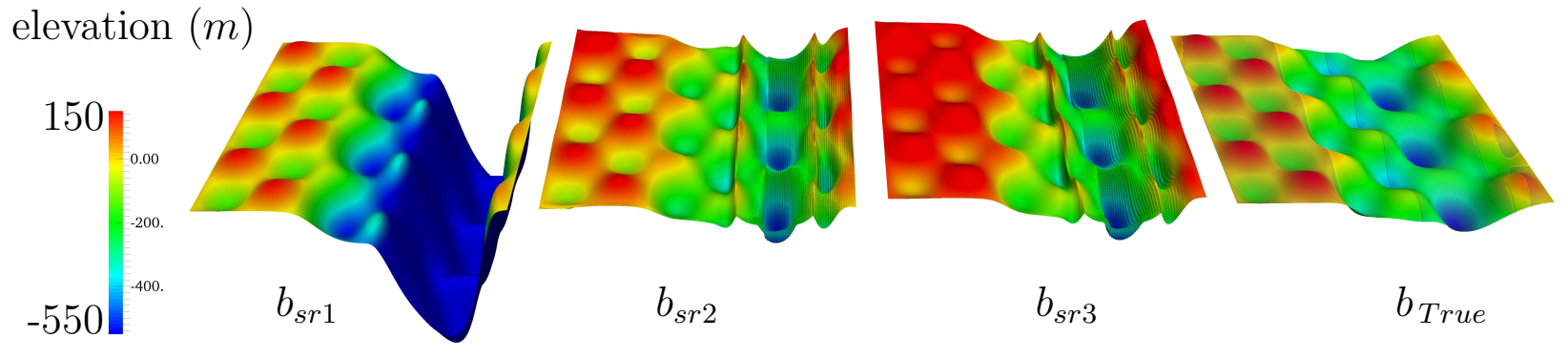

Figure 7: Test case 1 with exact measurements: bed elevation by areas, obtained after VDA\#1. (Down) From left to right : $h_{s r 1}, h_{s r 2}, h_{s r 3}$ and true value (with the lines indicating the sub-regimes areas defined by Table 1). (Top) From left to right : corresponding relative errors (for each $h_{s r}$ ). 


\begin{tabular}{|c|c|c|c|}
\hline Errors & First estimations & After VDA \#1 & After VDA \#2 \\
\hline \hline$\frac{\left\|h-h_{\text {true }}\right\|_{2}}{\left\|h_{\text {true }}\right\|_{2}}$ & 0.099 & 0.084 & 0.076 \\
\hline$\frac{\left\|h-h_{\text {true }}\right\|_{\infty}}{\left\|h_{\text {true }}\right\|_{\infty}}$ & 0.443 & 0.443 & 0.443 \\
\hline$\left\|H-H_{\text {obs }}\right\|_{\infty}$ & 14.08 & 0.15 & 0.19 \\
\hline$\frac{1}{n}\left\|H-H_{\text {obs }}\right\|_{2}$ & 0.0446 & 0.0002 & 0.0002 \\
\hline
\end{tabular}

Table 2: Test case \#1 with exact measurements: global errors (throughout the domain). (The errors on $H$ are obtained by running the direct model).

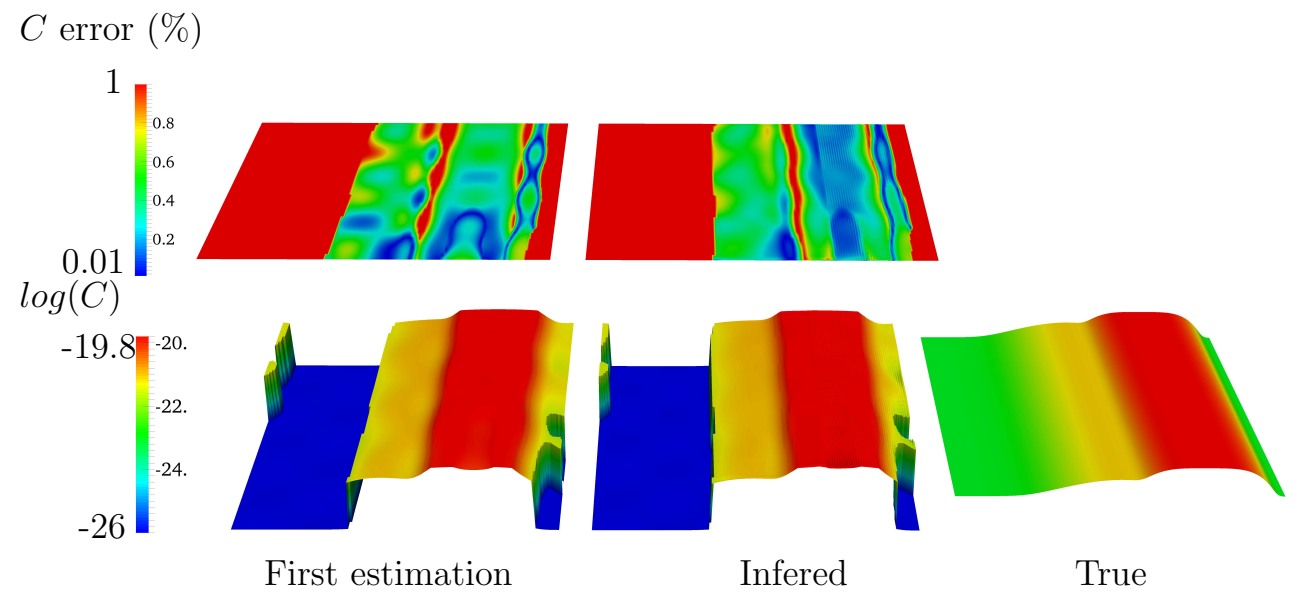

Figure 8: Test case 1 with exact measurements: friction coefficient $C$. (Down) From left to right: "1st guess" value resulting from $h^{(0)}$ and (15), inferred value after VDA\#1, true value. (Top) Corresponding relative errors.

The resulting friction field

Let us recall that with teh present inverse method the friction coefficient is a simple by-product of the depth value. In other words, the present method makes possible to separate the two crucial basal fields: the bed and the friction. Given a depth estimation, it is straightforward to compute the friction coefficient from (15). Numerically, both equalities give extremely close values. The resulting friction coefficient is plotted in Fig.8. The blue parts correspond to the sub-regime 1 areas. In these areas, a threshold has been applied (to a very low value); this threshold being defined such that $C<<A h$. Then the relative error in the sub-regime 1 is meaningless. In the sub-regime 2 area (resp. sub-regime 3), the error is globally between $10 \%$ and $60 \%$, excepted where the depth error is higher. Then at the sub-regime limits (stiff variations of $C$ of few order of magnitude), the error on the friction field can reach $100 \%$.

As expected, the estimated friction is accurate where the estimated depth is accurate. On the contrary where the estimated depth is less accurate, the estimated friction coefficient can't be accurate since it is implicitly set such that it makes fit the model outputs with the observations. Somehow this friction coefficient absorbs all errors (including the model errors which are null in the present twin experiments).

\subsubsection{VDA\#2: smoothing process}

The first VDA process (VDA\#1) was deliberately taking into account the observational term only. The goal was to analyse the inference capabilities of the present mix analytical-VDA method. From this VDA\#1 optimal solution $\left(\eta^{\# 1}, h^{\# 1}\right)$, a second VDA process is performed. The goal is to smooth the solution while keep fitting the data. The control variable is still $\omega=\ln (\eta)$. The cost function $j(\omega)$ is defined by (16) with $\alpha_{H}=1$ and $\alpha_{\text {reg }}=20$. The regularizing weight parameter $\alpha_{\text {reg }}$ is set following an empirical L-curve criteria, see e.g. [14]. Both the regularization term and the misfit term decrease during the optimization process. The resulting fields are plotted in Fig. 9 and Tab. 2. This second VDA process makes decrease the errors in the stiff variation areas (the "error peaks" located at the sub-regimes boundaries). Indeed, the error peaks on $\eta$ decrease from $20 \%$ to 15\%. Moreover, the VDA\#2 slightly improves the accuracy everywhere else by a few \%; compare Fig. 9 and Fig. 9. 
$h$ error $(\%)$

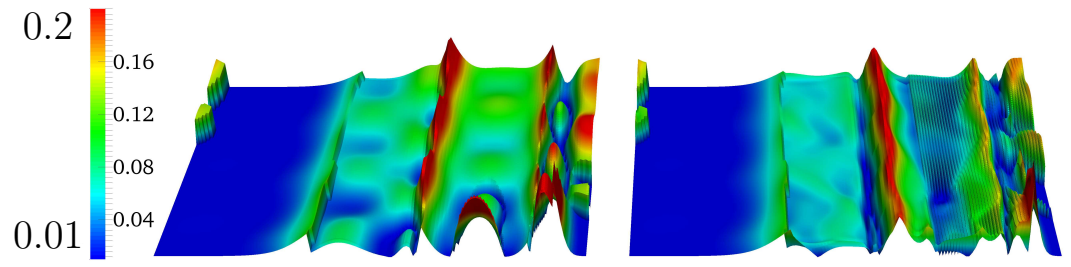

Bed elevation $(m)$

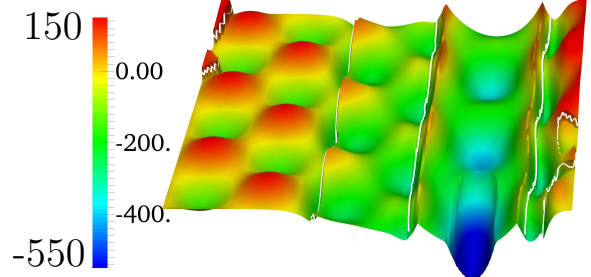

First estimation

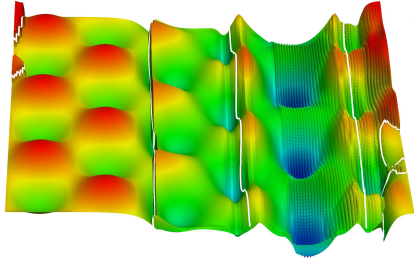

Infered

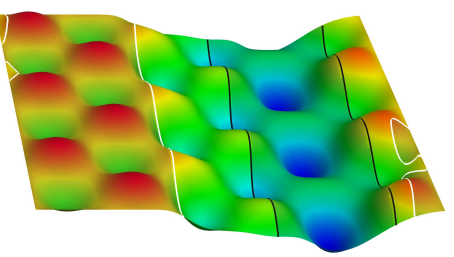

True

Figure 9: Test case 1 with exact measurements: bed elevation. (Down) From left to right: "1st guess" = value from (19), inferred value after VDA\#2, true value. (Top) Corresponding relative errors. The white lines denotes the sub-regime area boundaries defined from Table 1.

\subsection{Uncertain surface measurements: (quasi-)explicit sensitivities}

The surface measurements are the surface elevation $H$ (potentially acquired by altimetry) and the surface velocity $\mathbf{u}_{H}$ (potentially acquired by InSAR techniques). From these measurements, the slopes $\mathcal{S}$ and the observational term $\mathcal{Q}_{H}$ defined by (8) are estimated. The uncertainty sources are numerous and the resulting accuracy on $\mathcal{Q}_{H}$ depends on many factors. The error measurements on $H$ may be considered uniform in space; on the contrary the error measurements on $\left|\mathbf{u}_{H}\right|$ depends on its amplitudes, hence depending on the sub-regime areas. In other respect let us remark that the observational term $\mathcal{Q}_{H}$ is linear in $\mathbf{u}_{H}$ (hence introducing some noise in $\left|\mathbf{u}_{H}\right|$ is equivalent to introduce the same noise in $\left.\mathcal{Q}_{H}\right)$; on the contrary we have: $\mathcal{Q}_{H} \sim \mathcal{S}^{-q}$.

\subsubsection{The depth estimations $h_{s r} \square$ vs observational term perturbations $\delta \mathcal{Q}_{H}$}

Let us analyse the sensitivity of the three depth estimations $h_{s r} \square$ (the index $\square$ denoting the integer 1, 2 or 3) with respect to perturbations on $\mathcal{Q}_{H}$. The error measurements come independently from $\left|\mathbf{u}_{H}\right|$ or $\mathcal{S}$.

Note that concerning the slope values $\mathcal{S}$, an additional issue may be addressed: at what scale the numerical model has to be set up ? This classical slope scale questionning is difficult to tackle; however the answer (given in terms of length factor) is the same everywhere in the computational domain. From the sensitivity analysis below, a method to determine the correct slope scale of the xSIA model is presented in next section.

Let us denote by $h(1+\delta h)$ the resulting depth of a perturbed observational term $\mathcal{Q}_{H}(1+\delta \mathcal{Q})$. For each depth estimation, the resulting depth variation $\delta h$ is plotted vs $\delta \mathcal{Q}$ in Fig. 10(Left); for a $[-50,+50] \%$ perturbation range. In the sub-regime 1 case, it follows from (10) that: $\delta h_{s r 1}=(1+\delta \mathcal{Q})^{1 / q+1}-1$. Therefore the uncertainties on the observational term $\mathcal{Q}_{H}$, or equivalently on the surface velocity $\mathbf{u}_{H}$, is greatly damped, see Fig. 10 . A typical order of magnitude for these fully sheared flows (hence slow flows) is the following: a $30 \%$ noise on $\mathbf{u}_{H}$ (or equivalently on $\mathcal{Q}_{H}$ ) makes $h_{s r 1}^{\text {noise }}$ deviate from $h_{s r 1}$ by $\approx 8 \%$ only.

In the sub-regime 3 case, it follows from (14) that: $\delta h_{s r 3}=\frac{1}{(1+\delta \mathcal{Q})}-1$.

In the intermediate sub-regime 2 case, the dependency is more complex since the uncertainties propagate into the roots of polynomial (12). As a first step, the estimation (13) shows that if assuming the slip ratio $R_{s}$ invariant then the sensitivity of $h_{s r 2}$ with respect to $\mathcal{Q}_{H}$ is the same than those in the sub-regime 3 case: $\delta h=\frac{1}{(1+\delta \mathcal{Q})}-1$. Next to compute the complete sensitivity of $h_{s r 2}$ with respect to $\mathcal{Q}_{H}$, the polynomial (12) has to be numerically solved for each perturbed value of $\mathcal{Q}_{H}$. In Fig. 10 (Left), $\delta h_{s r 2}$ is plotted vs $\delta \mathcal{Q}$ for the few typical slip ratio values. To plot these curves, the different steps are the following: given a slip ratio $R_{s}$, the corresponding friction coefficient $C$ is evaluated from (6), next $\eta$ is evaluated from its expression (7), next $\mathcal{Q}_{H}$ is evaluated from (9), and finally the physical meaning root of the polynomial (12) with the perturbed $\mathcal{Q}_{H}$ is computed.

For weakly sheared - slipping flows (sub-regimes 2 and 3), hence relatively fast flows, a typical $10 \%$ noise on $\left|\mathbf{u}_{H}\right|$ makes deviate $h$ by $\approx 10-20 \%$, depending on the slip ratio value. Hence realistic uncertainties on the 

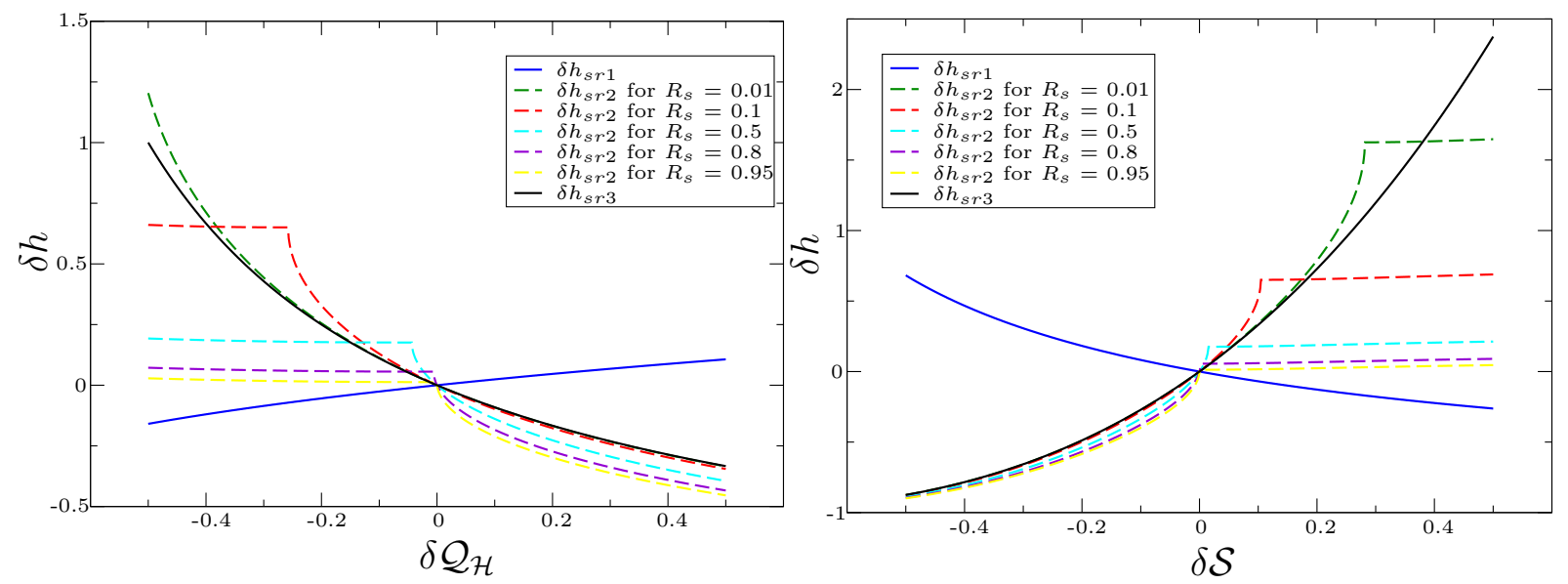

Figure 10: (Left) Explicit depth variations $\left(\delta h_{s r 1}, \delta h_{s r 2}, \delta h_{s r 3}\right):($ Left $)$ vs $\delta \mathcal{Q}_{H} ;$ (Right) vs $\mathcal{S}$. $\delta h_{s r 2}$ is obtained by computing the roots of the perturbed polynomial (12). The curves $\delta h_{s r 2}$ are plotted for typical slip ratio values.

surface velocity norm $\left|\mathbf{u}_{H}\right|$ does not prevent to estimate correctly the depth. In next section, a $[-20,+20] \%$ random noise (range indicated by the vertical dashed lines in Fig. 10 (Left)) will be added to $\mathcal{Q}_{H}$ before performing the complete inversion process (i.e. including the VDA process).

\subsubsection{The depth estimations $h_{s r \square}$ vs slope perturbations $\delta \mathcal{S}$}

If perturbing the slope $\mathcal{S}$ then $\mathcal{Q}_{H}$ is perturbed as follows: $\mathcal{Q}_{H}(1+\delta Q)=\frac{U_{H}}{\mathcal{S}^{q}(1+\delta S)^{q}}$. Therefore: $\delta Q=\frac{1}{(1+\delta S)^{q}}-1$ . Roughly a $[-10,+10] \%$ (resp. $[-20,+20] \%$ ) perturbation on $\mathcal{S}$ leads to a $[-40,+25] \%$ (resp. $[-50,+100] \%)$ perturbation on $\mathcal{Q}_{H}$. Now, let us compute the depth estimations $h_{s r} \square$ vs the slope perturbations $\delta \mathcal{S}$ (recall that the index $\square$ denotes the integer 1,2 or 3 ). As before, we denote by $h(1+\delta h)$ the resulting depth of the perturbed observational term $\mathcal{S}(1+\delta \mathcal{S})$.

In the sub-regime 1 case, the direct expression $\delta h_{s r 1}(\delta \mathcal{S})$ is straightforwardly obtained. In the sub-regime 3 case, $\delta h_{s r 3}$ depends on $\delta \mathcal{S}$ but also on $\delta \eta$.

In the sub-regime 2 case, the different steps are the following: given a slip ratio $R_{s}$ the corresponding friction coefficient $C$ is evaluated from (6), next $\eta$ is evaluated from (7), next $\mathcal{Q}_{H}$ is evaluated from (9), and finally the root $h_{s r 2}\left(1+\delta h_{s r 2}\right)$ of the polynomial (12) is computed (root corresponding to the perturbed value of $\mathcal{S}$ hence $\left.\mathcal{Q}_{H}\right)$.

In Fig. 10 (Right), the few $\delta h_{s r} \square$ values are plotted vs $\delta \mathcal{S}$. The difference of behavior between $\delta h_{s r 2}$ and $\delta h_{s r 1}\left(\right.$ resp. $\left.\delta h_{s r 3}\right)$ at high slip ratio value (resp. low value) is remarkable. In particular, for diminishing slopes values, $\delta h_{s r 2}$ and $\delta h_{s r 3}$ behaves similarly. For increasing slopes values, $\delta h_{s r 2}$ is more accurate than $\delta h_{s r 1}$ (for high slip ratio values).

\subsection{The complete inversion process with an uncertain observational term $\mathcal{Q}_{H}$}

In the previous numerical depth inference, the surface measurements were supposed to be exact. In the present numerical experiment, a $20 \%$ random noise is added to $\mathcal{Q}_{H}$. Recall that this perturbation in $\mathcal{Q}_{H}$ is equivalent to a $20 \%$ random perturbation in $\left|\mathbf{u}_{H}\right|$; it corresponds approximatively to a $5 \%$ random perturbation in $\mathcal{S}$ too, see Fig. 10. The consequences of this randomly perturbed $\mathcal{Q}_{H}$ to the inferred depth values are presented. As a first step, the randomly perturbed $\mathcal{Q}_{H}$ is smoothed by minimizing the following cost function:

$$
g\left(\mathcal{Q}_{H}\right)=\frac{1}{2}\left\|Q_{H}-\mathcal{Q}_{H}^{o b s}\right\|_{2, \Omega}^{2}+\frac{\alpha}{2}\left\|\nabla \mathcal{Q}_{H}\right\|_{2, \Omega}^{2} d x \equiv \frac{1}{2}\left[g_{1}\left(Q_{H}\right)+\alpha g_{2}\left(\nabla \mathcal{Q}_{H}\right)\right]
$$

Again the regularizing weight parameter $\alpha$ is set following an empirical L-curve criteria. This led to set: $\alpha=5.210^{11}$. The cost function values $\left(g_{1}\left(Q_{H}\right), g_{2}\left(\nabla \mathcal{Q}_{H}\right)\right)$ equals: $\left(0 ., 5.210^{11}\right)$ at the first iteration and $\left(1.810^{9}, 3.710^{10}\right)$ at the $32^{\text {nd }}$ (last) iteration. The corresponding values of $\mathcal{Q}_{H}$ are plotted in Fig. 11.

Next the VDA process to compute $\eta$ is performed. Let us notice that the noise on $\mathcal{Q}_{H}$ has a direct impact on the a-priori slip ratio map $R_{s}^{(0)}$ next on the first estimation $h^{(0)}$, next on the first guess $\eta^{(0)}$.

However, despite the introduction of noise on $\mathcal{Q}_{H}$, again the first estimation $h^{(0)}$ is an excellent estimation of the true depth (not plotted). Next, the complete VDA process is performed: the VDA\#1 process, $\alpha_{\omega}=0$ in (16), next the VDA\#2 process with $\alpha_{\omega}=7$. From the obtained optimal value $\eta^{*}$, the depth estimations are 
$\mathcal{Q}_{\mathcal{H}}$ error $(\%)$

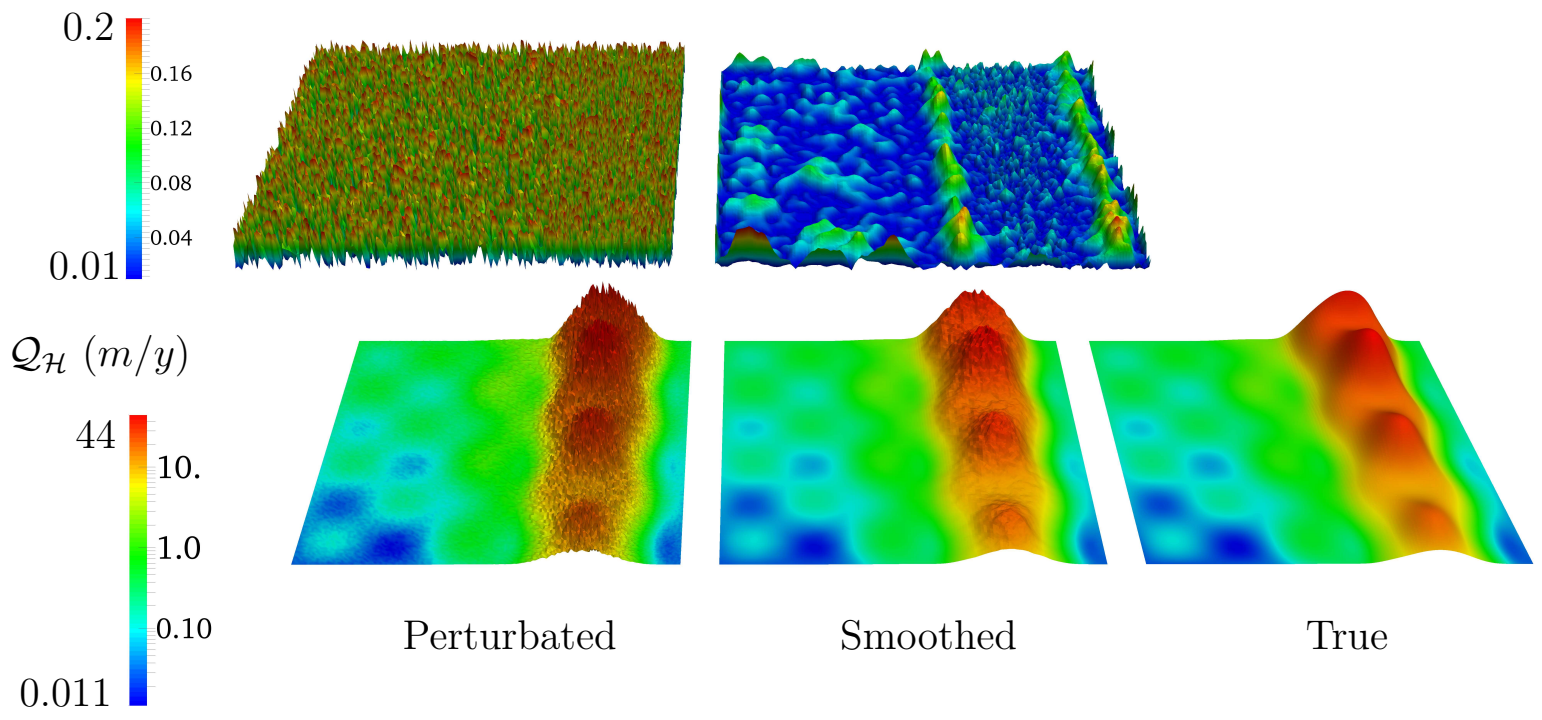

Figure 11: Test case 1 with uncertain measurements $\mathcal{Q}_{H}$. (Down) From left to right: $\mathcal{Q}_{H}$ with noise, after smoothing process, original "true" field. (Top) The corresponding relative errors.

\begin{tabular}{|c|c|c|c|}
\hline Errors & First estimation & After VDA \#1 & After VDA \#2 \\
\hline \hline$\frac{\left\|h-h_{\text {true }}\right\|_{2}}{\left\|h_{\text {true }}\right\|_{2}}$ & 0.089 & 0.079 & 0.075 \\
\hline$\frac{\left\|h-h_{\text {true }}\right\|_{\infty}}{\left\|h_{\text {true }}\right\|_{\infty}}$ & 0.533 & 0.468 & 0.437 \\
\hline$\left\|H-H_{\text {obs }}\right\|_{\infty}$ & 8.294 & 0.26 & 0.27 \\
\hline$\frac{1}{n}\left\|H-H_{\text {obs }}\right\|_{2}$ & 0.0264 & 0.0004 & 0.0003 \\
\hline
\end{tabular}

Table 3: Test case \#1 with uncertain measurements: global errors (throughout the domain). (The errors on $H$ are obtained by running the direct model).

computed. The resulting bed elevation is plotted in Fig. 12, see also Tab. 3. The three depth estimates $h_{s r}^{(0)}$ give results roughly as accurate as in the exact measurements case. Indeed $h_{s r 1}$ is accurate to less than $5 \%$ in the sub-regime 1 area; $h_{s r 2}$ (resp. $h_{s r 3}$ ) is accurate to $10 \%$ (and less) in the inner part of the sub-regime 2 area

(resp. sub-regime 3 area) and accurate to $20 \%$ at the area boundaries. Compared to the first depth value $h^{(0)}$, the VDA process improved the solution mainly in the sub-regime 3 area: the error decreases from $10-30 \%$ before VDA, to $5-20 \%$ after VDA, Fig. 12 .

The resulting friction field. Given the depth $h$ everywhere, the friction coefficient $C$ is obtained explicitly by evaluating (15), both formula giving quite the same values. The resulting friction coefficient is not plotted since its patterns and its accuracy are very similar to the case of exact measurements, see Fig.8.

In summary, a $20 \%$ random noise on $\mathcal{Q}_{H}$ throughout the computational domain (potentially corresponding to a $20 \%$ noise on $\left|\mathbf{u}_{H}\right|$ or to a $5 \%$ noise on $\mathcal{S}$ ) does not affect the robustness of the present inversion approach nor the accuracy of the inferred depth values. Nevertheless this encouraging result has to be tempered since the slope term $\mathcal{S}$ considered in the xSIA equation (7) was considered as exact. Only the observational term $\mathcal{Q}_{H}$ (appearing throughout the complete inversion process: explicit formulas, first guesses and the VDA process) has been perturbed. The questioning of the slope uncertainty (and/or the slope scale definition) is addressed in the last section.

\section{Case 2: lateral measurements}

The present numerical experiment is the same as the previous one with exact data but the location of the depth measurements. In the present case, the depth is measured following the lateral line/track indicated in blue in Fig. 3. Consequences are important since the depth measurements are available in a mono-regime area only 
$h$ error $(\%)$
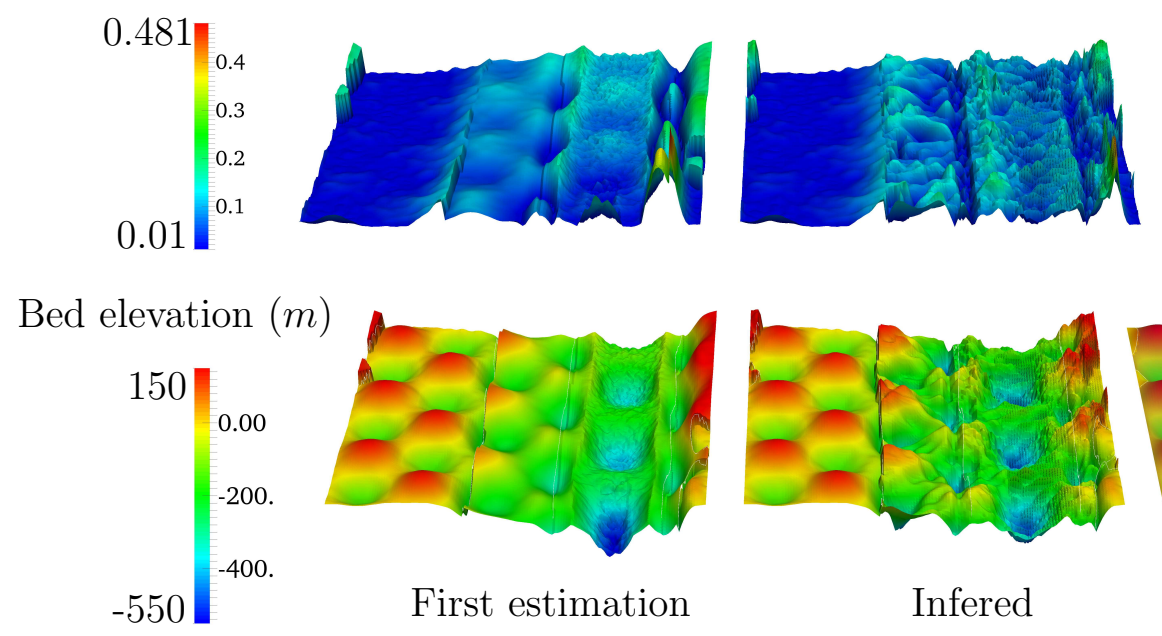

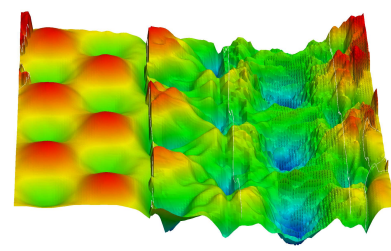

Infered

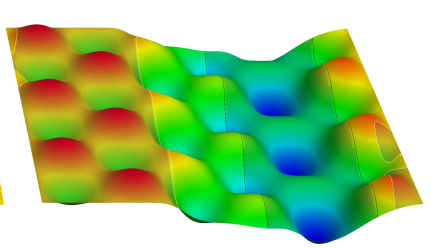

True

Figure 12: Test case 1 with uncertain measurements (20\% random noise on $\mathcal{Q}_{H}$ ): bed elevation (in $\mathrm{m}$ ). (Down) From left to right: 1st estimation = the value infered from (19) after the smoothing process, inferred value after VDA, true value (with the lines indicating the sub-regimes areas defined by Table 1). (Top) Corresponding relative errors. The white lines denotes the different regime zones.

(fully sheared area) furthermore parallel to a flow line. In particular, these measurements do not include any point in the canyon nor various upstream points. Actually, the present measurement track is almost equivalent to a single location measurement, see the blue dots in Fig. 4 (Right). The present configuration makes the inverse problem particularly challenging.

\subsection{A-priori slip ratio map $R_{s}^{(0)}$ and first estimation $h^{(0)}$}

The first step of the inverse method consists to define the a-priori slip ratio map $R_{s}^{(0)}$. In the present configuration, the depth measurements do not include a varying slip ratio, hence extra a-priori have to be made. Then as already mentioned the two a-priori are made:

- a surface velocity $\left|\mathbf{u}_{H}\right|=1 \mathrm{~m} / y$ corresponds to slip ratio $R_{s}=0.5$,

- a surface velocity $\left|\mathbf{u}_{H}\right|=20 \mathrm{~m} / y$ corresponds to slip ratio $R_{s}=0.03$,

see the two green points in Fig. 4. Given the resulting a-priori slip ratio map $R_{s}^{(0)}$, the first depth estimation $h^{(0)}$ is computed, next the first guess $\eta^{(0)}$, as previously described. These first values / guesses are plotted in Fig. 13, see also Tab. 4. Again, the first depth estimation $h^{(0)}$ is already excellent.

Let us point out that if the a-priori slip ratio $R_{s}^{(0)}$ is really bad, typically by considering the second point equal to $(2 \mathrm{~m} / \mathrm{y}, 0.03)$, see Fig. 4 Right, then the first bed estimation $b^{(0)}$ is bad too, following $(19): h^{(0)} \sim R_{s}^{\frac{1}{(q+1)}}$. However in such a case, the VDA process improves more the first estimation $h^{(0)}$ and leads to quite a good depth value. Typically the 2-norm relative error of the final depth estimation $h$ is approximatively $20 \%$ instead of $7-8 \%$, see Tab. 4 .

\subsection{Inferred bed elevation after VDA}

Next the VDA process is performed from the first guess value $\eta^{(0)}$. Since the data are exact, the regularization term $j_{\text {reg }}$ is not really necessary, see Tab. 4 . Then the presented results are those of VDA\#1 only: $\alpha_{\text {reg }}=0$ in (16). The minimization process converges in 30 iterations, reaching the $+/-10 \mathrm{~cm}$ uncertainties on the surface elevation. Next from the obtained optimal value $\eta^{*}$, the depth estimations are computed. Next, following the a-priori definitions of the sub-regime areas, Table 1 , the combination of the 3 estimations $h_{s r} \square$ gives the inferred bed plotted in Fig. 14 .

Like in Case 1, at the sub-regime limits (corresponding to the large and stiff variations of $\eta$ ), the relative error is higher (approximatively $20 \%$ in $h$ ) than anywhere else. Out of these stiff regime transition areas, the error 


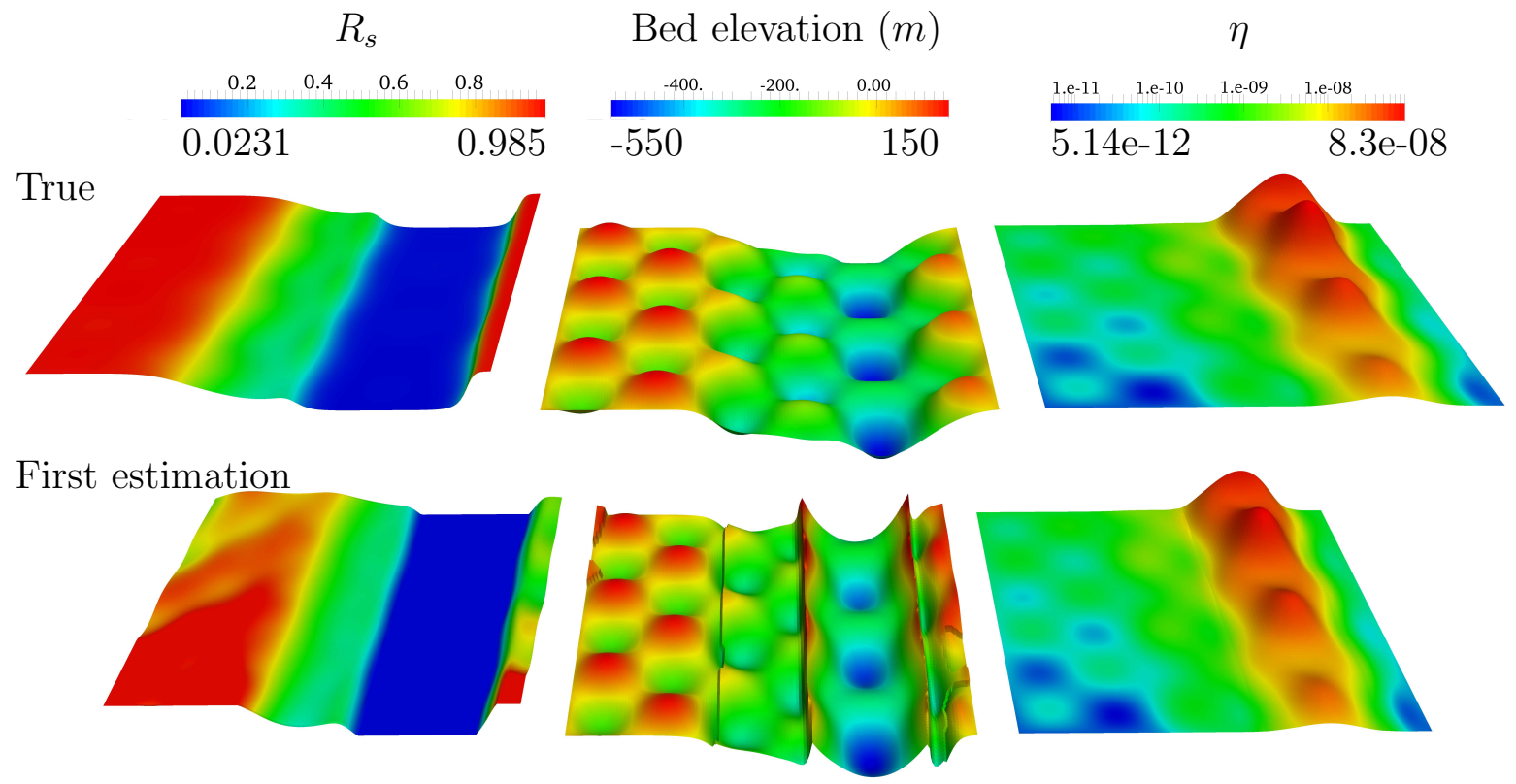

Figure 13: Test case 2 (lateral measurements). From left to right : slip ratio $R_{s}$, bed elevation $b$ (in $\mathrm{m}$ ), effective diffusivity $\eta$ (log scale). (Top) True values. (Down) a-priori values $R_{S}^{(0)}$, 1 st bed estimation $b^{(0)}$ infered from (19), and the resulting value $\eta^{(0)}$ from (12) (1st guess value for the VDA process).

\begin{tabular}{|c|c|c|c|}
\hline Errors & First estimation & After VDA \#1 & After VDA \#2 \\
\hline \hline$\frac{\left\|h-h_{\text {true }}\right\|_{2}}{\left\|h_{\text {true }}\right\|_{2}}$ & 0.084 & 0.076 & 0.070 \\
\hline$\frac{\left\|h-h_{\text {true }}\right\|_{\infty}}{\left\|h_{\text {true }}\right\|_{\infty}}$ & 0.443 & 0.443 & 0.446 \\
\hline$\left\|H-H_{\text {obs }}\right\|_{\infty}$ & 6.89 & 0.21 & 0.17 \\
\hline$\frac{1}{n}\left\|H-H_{\text {obs }}\right\|_{2}$ & 0.0236 & 0.0002 & 0.0002 \\
\hline
\end{tabular}

Table 4: Test case \#2: global errors (throughout the domain). (The errors on $H$ are obtained by running the direct model).

on the depth $h$ is small, lower than 7\%; even lower in the sub-regime 1 area. Again the three estimates give accurate results in their respective domain of validity. Again if comparing with the first estimations, Fig. 13, the VDA process improves a bit the depth estimates in the inner parts of sub-regimes 2 and 3 and at the "sub-regimes boundaries" (making decrease the peak errors).

Concerning the friction coefficient $C$ (which is computed a-posteriori), its accuracy is similar to the case of downstream measurements, see Fig.8. For this field $C$, the VDA process improves greatly its accuracy, in particular in the sub-regime 2 area. Typically the VDA process makes decrease the errors from $30 \%$ to less than $20 \%$ in the smooth areas; and from approximatively $200 \%$ to $100 \%$ in the stiff transition areas.

On the robustness of the present inverse method The present depth inference is (slightly) more accurate than in the downstream measurements case (test case 1). This result is only due to the better a-priori slip ratio law $R_{s}^{(0)}$, see the fitting law in Fig. 4 (Right). More importantly this test case 2 demonstrates the following important feature: since the present depth inference is based on an elliptic model, and not a hyperbolic one, the depth measurement locations are not crucial. Typically, measuring the depth at upstream, downstream or laterally, does not affect the inversion method accuracy. The important starting points of the present approach are the accuracy of the surface observations (the observational term $\mathcal{Q}_{H}$ ) and the accuracy of $R_{s}^{(0)}$ derived from the depth measurements. 
0.2

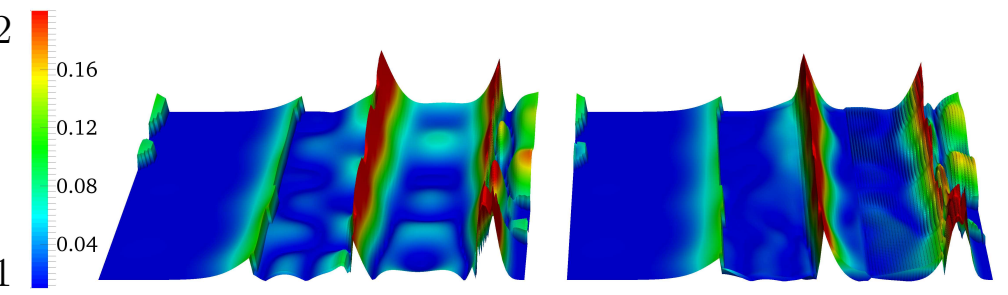

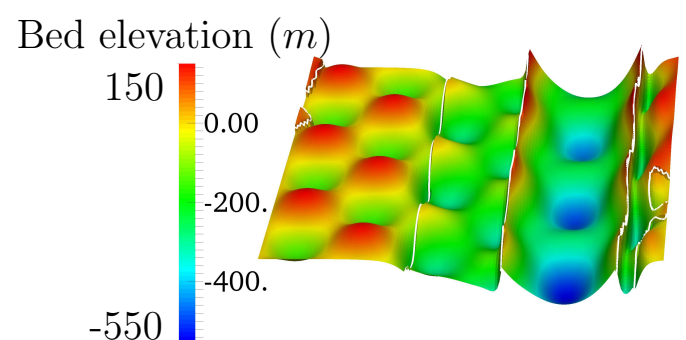

First estimation

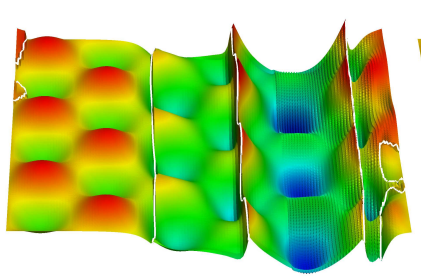

Infered

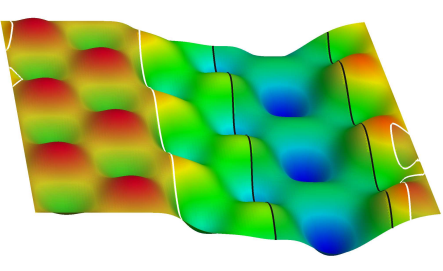

True

Figure 14: Test case 2: bed elevation. (Down) From left to right: first estimation from (19), inferred value after VDA, true value. (Top) Corresponding relative errors.

The white lines denotes the sub-regime area boundaries defined from Table $1 .$.

\section{Inference of the correct slope scale in the xSIA model}

As already discussed, a major difficulty to set up a SIA model is to manage its high sensitivity to the slope values

$\mathcal{S}$. This sensitivity means sensitivity with respect to the slope error measurements but also with respect to the slope scale definition of the flow model. This is a global concept in space. Typically for the ice-sheets, out of ice-streams and/or far inland to be sub-regime 1 compatible, a standard accepted slope scale is approximatively $10 h^{*}, h^{*}$ being a characteristic thickness. Nevertheless, for more sliding flows (i.e. in sub-regime 2 and subregime 3 areas), this characteristic scale may have to be adapted, see the complimentary analyses [3, 11, 25]. From the present depth estimations, a method to estimate the slope scale representing correctly the surface data with the xSIA model (7) can be derived.

From data basis. A pure data-oriented step would consist to infer correlations between the slope scale and the depth from the available depth measurements (e.g. measurements based on the blue or red lines in Fig. 3 or based on the massive data sets available for the two ice-sheets[35], [2]). Indeed, from a data set providing all the model outputs (surface elevation, surface velocity and depth in particular), it is possible to infer the corresponding xSIA slope scale. Then, given the numerical model (including the computational and bed topography grids), this optimal slope scale can be considered throughout each sub-regime areas. (Indeed, the low-band filtering feature of the flow depends on the flow regime, see e.g. [25]).

From the present depth estimations. A complimentary method would rely on the few present depth estimations. First let us recall that the sub-regime 2 estimation, see (12) and (13), is valid everywhere i.e. it is valid in the three sub-regime areas (despite it may be not the most accurate estimation in sub-regime 1 areas since numerical errors in $R_{s}$ or $\eta$, see the previous discussion). Then the correct xSIA model slope scale can be defined from the radically different behaviors of the depth estimations with respect to $\mathcal{Q}_{H}$. Indeed, the sub-regime 1 depth estimation (10) behaves like $\mathcal{Q}_{H}^{1 /(q+1)}$ while the sub-regime 2 estimation (13) behaves in $\mathcal{Q}_{H}^{-1}$. Therefore, given all the points located in the sub-regime 1 and sub-regime 2 areas (point set identified from a-priori values like Tab. 1 or from the a-priori slip ratio value $R_{s}^{(0)}$ ), the two depth estimations can be computed from the observed slope value at different slope scales, typically at $2^{k}$ characteristic depth and/or mesh size, with $k=0, . ., 3$.

Then the correct slope scale (which should be an uniform concept for each sub-regime area) is those giving the closest depth obtained from the two estimations. In other words, the correct scale corresponds to the intersection point between the $h_{s r 1}$ and $h_{s r 2}$ curves in Fig. 10. In a large set of data, the intersection point can be statistically estimated.

Since the present depth estimations are local, this method to infer the optimal / correct slope scale of the xSIA model can be illustrated for 1D uniform flows without any loss of generality. 

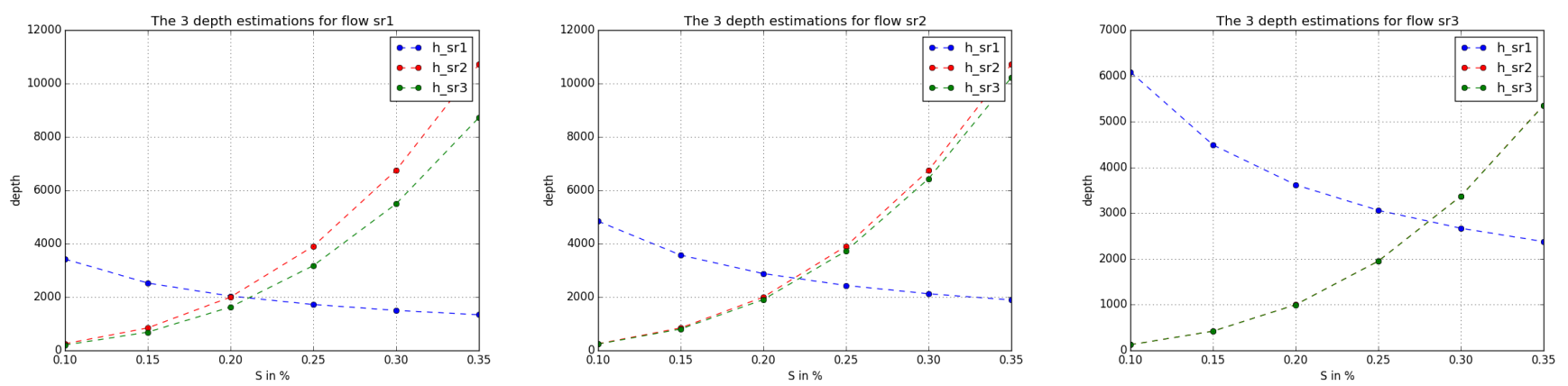

Figure 15: $1 \mathrm{D}$ uniform flows: the three depth estimations $h_{s r} \square$. From left to right: sub-regime 1 case, sub-regime 2 case, sub-regime 3 case.

Illustration with 1D uniform flows. Let us consider 1D uniform flows. The fluid properties $(A, q, \rho)$ are identical as previously and three typical flows are considered. For all three, the slope value is $\mathcal{S}=0.2 \%$.

Each flow differs from each other by the values of $\mathbf{u}_{H}$ and $h$. Then are evaluated: the observational term $\mathcal{Q}_{H}$, the friction coefficient $C$ from (15), the effective diffusivity $\eta$ and the slip ratio $R_{s}$. Finally, the three resulting curves are plotted in Fig. 15. In Fig. 15 (Right), the graphs of $h_{s r 2}$ and $h_{s r 3}$ are fully superposed. This curves illustrate the radically different behaviors of the explicit depth estimations with respect to $\mathcal{S}$. the intersection point between the two estimations $h_{s r 1}$ and $h_{s r 2}$, see Fig. 15 (Left). In the sub-regime 2 and sub-regime 3 areas, the situation is a bit different. The sub-regime 1 estimation (10) over estimates the true value (as soon as the sliding becomes quite important). Then the intersection points give an upper-bound only of the correct depth, hence an upper-bound only of the correct slope value, see Fig. 15 (Middle) and (Right).

In other words, the present three depth estimations make possible to infer the correct slope scale to set up consistently the xSIA models, if some depth measurements are available. Indeed, the approach detailed here in $1 \mathrm{D}$ can be applied in the 2D case since the estimations are point-wise. Then the inference of the correct slope 
scale from available complete data (including depth measurements) in the weakly sheared areas (sub-regime 1 areas) becomes possible. Next this optimal slope scale can be applied in the xSIA model throughout the computational domain i.e. where depth data are not available and in the other sub-regime areas.

\section{Conclusion}

This study proposes a new inverse method to infer the bottom topography $b$ and the friction coefficient $C$ in the Shallow Ice Approximation (SIA) models. Then the method can be applied to the great majority of the ice sheet surfaces (sheared flow areas) and to many high mountain glaciers. In a mathematical point of view, the present inverse method can be applied to any shallow sheared generalized Newtonian fluid flow, for example lava flows (with the thermal field given), mud flows and many polymer flows. The method is based on the definition of sub-regimes depending on the slip ratio, different combinations of explicit field expressions with a linear-quadratic optimal control problem solved by Variational Data Assimilation (where the direct equation is a gentle linear elliptic equation). This leads to a particularly robust method even in presence of uncertain surface measurements and independently of the depth measurement locations (on the contrary if inverting the mass equation only since it is a first order transport equation); also the potential various error measurements are damped (and not propagated). Finally the difficult question aiming at determining the correct slope scale in a SIA model may be solved by cross-comparing the few depth estimations proposed. While assessing the method with few difficult academic multi-regime test cases, the only method drawback turned out to be its relatively low accuracy in the stiff variation areas of the friction coefficient $C$ (typically in canyon margins where $C$ varies of few orders of magnitude). However this demonstrates the method capability to infer a reliable bottom topography elevation $b$ despite an extremely varying friction coefficient $C$. This new inverse method can be straightforwardly extended to unsteady flows if the available surface observations are time-dependent too (assuming that the initial condition is either not important in the considered time scale or can be efficiently infered). This promising new inverse method will be assessed with real data sets in a forthcoming study.

Acknowledgements The first author is deeply grateful to E. Larour, M. Morlighem, E. Rignot and H. Seroussi from JPL/Caltech-NASA and Univ. California of Irvine for co-funding him in 2014. The discussions the author had in this group greatly contributed to his understanding of remote-sensed data of glaciers.

The second author has been funded by CNES TOSCA grant (forthcoming NASA-CNES SWOT mission) during his INSA Master's degree internship, making possible the 2D Python/FEniCS computational code development used in this article.

Finally the authors acknowledge the three anonymous reviewers which have read the article into details and made improved its readibility.

\section{References}

[1] M. Alnæs, J. Blechta, J. Hake, A. Johansson, B. Kehlet, A. Logg, C. Richardson, J. Ring, M. Rognes, E. Wells, and N. Garth. The fenics project version 1.5. Archive of Numerical Software, 3(100):9-23, 2015.

[2] J. Bamber, J. Griggs, R. Hurkmans, J. Dowdeswell, S. Gogineni, I. Howat, J. Mouginot, J. Paden, S. Palmer, E. Rignot, et al. A new bed elevation dataset for greenland. The Cryosphere, 7(2):499-510, 2013.

[3] K. Barclay and K. Echelmeyer. Stress-gradient coupling in glacier flow: I. longitudinal averaging of the influence of ice thickness and surface slope. Journal of Glaciology, 32(111):267-284, 1986.

[4] M. Boutounet, J. Monnier, and J.-P. Vila. Multi-regime shallow free surface laminar flow models for quasi-newtonian fluids. European Journal of Mechanics-B/Fluids, 55:182-206, 2016.

[5] A.-L. Dalibard and D. Gérard-Varet. Effective boundary condition at a rough surface starting from a slip condition. Journal of Differential Equations, 251(12):3450-3487, 2011.

[6] A. Fowler. Mathematical geoscience, volume 36. Springer, 2011.

[7] P.-A. Garambois and J. Monnier. Inference of effective river properties from remotely sensed observations of water surface. Advances in Water Resources, 79:103-120, 2015. 
[8] D. Gilbarg and N. Trudinger. Elliptic partial differential equations of second order. springer, 2015.

[9] R. Glowinski and P. Le Tallec. Augmented Lagrangian and operator-splitting methods in nonlinear mechanics, volume 9. SIAM, 1989.

[10] R. Greve and H. Blater. Dynamics of Ice Sheets and Glaciers. Advances in Geophysical and Environmental Mechanics and Mathematics. 2009.

[11] G. Gudmundsson. Transmission of basal variability to a glacier surface. Journal of Geophysical Research: Solid Earth, 108(B5), 2003.

[12] G. Gudmundsson. Analytical solutions for the surface response to small amplitude perturbations in boundary data in the shallow-ice-stream approximation. The Cryosphere, 2(2):77-93, 2008.

[13] G. Gudmundsson and M. Raymond. On the limit to resolution and information on basal properties obtainable from surface data on ice streams. The Cryosphere Discussions, 2(3):413-445, 2008.

[14] P. Hansen and D. Prost O'Leary. The use of the l-curve in the regularization of discrete ill-posed problems. SIAM Journal on Scientific Computing, 14(6):1487-1503, 1993.

[15] C. Heining and M. Sellier. Direct reconstruction of three-dimensional glacier bedrock and surface elevation from free surface velocity. AIMS Geosciences, 2:45-63, 2016.

[16] M. Honnorat, J. Monnier, N. Rivière, É. Huot, and F.-X. Le Dimet. Identification of equivalent topography in an open channel flow using lagrangian data assimilation. Computing and visualization in science, 13(3):111-119, 2010.

[17] M. Huss and D. Farinotti. A high-resolution bedrock map for the antarctic peninsula. The Cryosphere, 8(4):1261-1273, 2014.

[18] K. Hutter. Theoritical Glaciology. 1983.

[19] G. Jouvet and E. Bueler. Steady, shallow ice sheets as obstacle problems: well-posedness and finite element approximation. SIAM Journal on Applied Mathematics, 72(4):1292-1314, 2012.

[20] G. Jouvet, J. Rappaz, E. Bueler, and H. Blatter. Existence and stability of steady-state solutions of the shallow-ice-sheet equation by an energy-minimization approach. Journal of Glaciology, 57(202):345-354, 2011.

[21] E. Larour, J. Utke, A. Csatho, B. andSchenk, H. Seroussi, M. Morlighem, E. Rignot, N. Schlegel, and A. Khazendar. Inferred basal friction and surface mass balance of the northeast greenland ice stream using data assimilation of icesat (ice cloud and land elevation satellite) surface altimetry and issm (ice sheet system model). Cryosphere, 8(6), 2014.

[22] F.-X. LeDimet and O. Talagrand. Variational algorithms for analysis and assimilation of meteorological observations: theoretical aspects. Tellus A, 38(2):97-110, 1986.

[23] A. Logg, G. Wells, and J. Hake. Dolfin: A c++/python finite element library. In Automated Solution of Differential Equations by the Finite Element Method, pages 173-225. Springer, 2012.

[24] N. Martin and J. Monnier. Adjoint accuracy for the full-stokes ice flow model: limits to the transmission of basal friction variability to the surface. The Cryosphere, 8:721-741, 2014.

[25] N. Martin and J. Monnier. Inverse rheometry and basal properties inference for pseudoplastic geophysical flows. European Journal of Mechanics-B/Fluids, 50:110-126, 2015.

[26] L. Michel, M. Picasso, D. Farinotti, A. Bauder, M. Funk, and H. Blatter. Estimating the ice thickness of mountain glaciers with an inverse approach using surface topography and mass-balance. Inverse Problems, 29(3):035002, 2013.

[27] L. Michel, M. Picasso, D. Farinotti, M. Funk, and H. Blatter. Estimating the ice thickness of shallow glaciers from surface topography and mass-balance data with a shape optimization algorithm. Computers Ef Geosciences, 66:182-199, 2014.

[28] J. Monnier. Variational data assimilation: from optimal control to large scale data assimilation. Open Online Course (videos) with the accompanying manuscript (143 pages). INSA - University of Toulouse, 2014. 
[41] M. Sellier. Inverse problems in free surface flows: a review. Acta Mechanica, 227(3):913-935, 2016

[42] M. Sellier, A. Gessese, and C. Heining. Analytical and numerical bedrock reconstruction in glacier flows from free surface elevation data. 2012.

[43] T. Thorsteinsson, C. Raymond, G. Gudmundsson, Robert. Bindschadler, P. Vornberger, and I. Joughin. Bed topography and lubrication inferred from surface measurements on fast-flowing ice streams. Journal of Glaciology, 49(167):481-490, 2003.

[44] R. Warner and W. Budd. Derivation of ice thickness and bedrock topography in data-gap regions over antarctica. Annals of Glaciology, 31(1):191-197, 2000. 